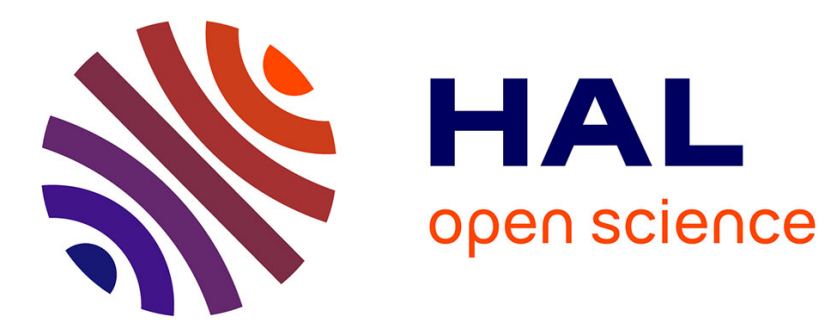

\title{
Regional Differences in Job Satisfaction
}

Richard Jones, Peter Sloane

\section{To cite this version:}

Richard Jones, Peter Sloane. Regional Differences in Job Satisfaction. Applied Economics, 2009, 41 (08), pp.1019-1041. 10.1080/00036840601019067 . hal-00581972

\section{HAL Id: hal-00581972 \\ https://hal.science/hal-00581972}

Submitted on 1 Apr 2011

HAL is a multi-disciplinary open access archive for the deposit and dissemination of scientific research documents, whether they are published or not. The documents may come from teaching and research institutions in France or abroad, or from public or private research centers.
L'archive ouverte pluridisciplinaire $\mathbf{H A L}$, est destinée au dépôt et à la diffusion de documents scientifiques de niveau recherche, publiés ou non, émanant des établissements d'enseignement et de recherche français ou étrangers, des laboratoires publics ou privés. 


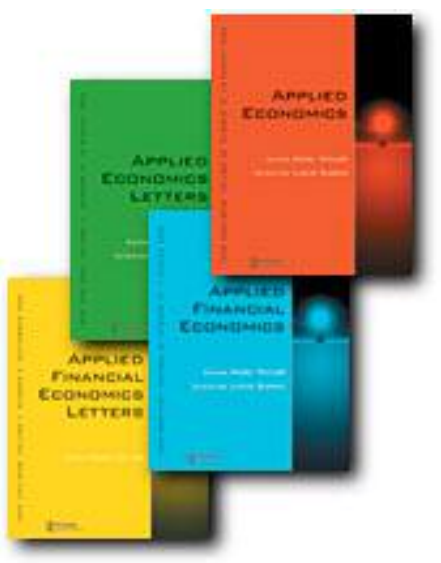

\section{Regional Differences in Job Satisfaction}

\begin{tabular}{|r|l|}
\hline Journal: & Applied Economics \\
\hline Manuscript ID: & APE-05-0425.R1 \\
\hline Journal Selection: & Applied Economics \\
\hline JEL Code: & $\begin{array}{l}\text { J28 - Safety } \mid \text { Accidents } \mid \text { Industrial Health|Job Satisfaction } \mid \text { Related } \\
\text { Public Policy }<, \text { R10 - General < }\end{array}$ \\
\hline Keywords: & job satisfaction, regional labour markets \\
\hline
\end{tabular}

\section{\scholarONE" \\ Manuscript Central}




\title{
Regional Differences in Job Satisfaction
}

\author{
Richard J Jones and Peter J Sloane ${ }^{1}$ \\ WELMERC, Department of Economics, University of Wales Swansea \\ September 2006
}

\begin{abstract}
Job satisfaction is significantly higher in Wales than in London and the South East, the rest of England and Scotland. This is despite the fact that among these four regions, earnings are lowest in Wales. Using data from the British Household Panel Survey (BHPS), we investigate the determinants of job satisfaction and attempt to explain why workers in Wales are happier in their work than workers in other parts of the UK. We find that workers in Wales appear to be less concerned about pay than workers in other regions. We suggest that because lower earnings tend to be associated with higher levels of unemployment and inactivity, being in work may be regarded more favourably in more economically depressed regions. We also suggest the climate of industrial relations, as perceived by workers, is better in Wales than elsewhere.
\end{abstract}

Keywords: Job satisfaction, Wales, regional labour markets.

\footnotetext{
${ }^{1}$ Please direct all correspondence to this author at the following address: WELMERC, Department of Economics, University of Wales Swansea, James Callaghan Building, Singleton Park, Swansea, SA2 8PP, UK, phone (44) 01792513 319, fax (44)1792 295872, e-mail p.j.sloane@swansea.ac.uk. Sloane is also Research Fellow, IZA, Bonn.
} 


\section{INTRODUCTION}

In recent years economists have used worker reported job satisfaction to examine the utility obtained from work. Standard micro-economic theory suggests there is a trade-off between earnings and hours of work, with satisfaction rising as wage income increases and decreasing as hours of work rise. However, there is evidence to suggest that these features are not the only or even the most important determinants of job satisfaction. In particular, individuals obtain satisfaction from the nature of work itself, from feelings of job security, from relationships with coworkers and much else besides. (Clark 2001). Both absolute pay and relative pay have been found to influence job satisfaction as workers feelings of job equity are governed not only by what they earn but also by what other workers in similar positions earn. (Rees 1993, Baxter 1973 and 1993). There are also differences between men and women in the levels of reported satisfaction and their determinants, with women being less driven by pay than is the case with men. (Sloane and Williams 2000)

Among previous papers in this journal examining aspects of job satisfaction Idson (1990) has focused on establishment size, Meng (1990) on trade unions, Belfield and Harris (2002) on education, Brown and McIntosh (2003) on the low wage service sector and Gazioglu and Tansel (2006) on a range of individual and job-related factors. Another group of papers has focused on differences in job satisfaction across nations. Thus Sousa-Poza and Sousa-Posa (2000) compared 21 countries using data from the 1997 International Social Survey Program. They found that levels of job satisfaction varied across countries and these differences could be partly attributed to differences in work role inputs and outputs. Further, while some 
determinants of job satisfaction, such as having an interesting job and good relations with management, were important in all countries, others such as pay and job security tended to be country specific. There are also gender differences in job satisfaction across country with women reporting significantly higher job satisfaction than men in the UK, the US, Hungary and New Zealand, but significantly lower satisfaction in some other countries, notably Spain. (See Sousa-Poza and SousaPosa, 2000). Diaz-Serrano and Cabral Vieira (2005) examined the difference in job satisfaction between lower and higher paid workers within the European Union using the European Union Community Household Panel data over the period 1994-2001. They find that low paid workers report a significantly lower level of job satisfaction than higher paid workers in most countries, but that the reverse applies in the UK. Green and Tsitsianis (2005) attempt to explain trends in job satisfaction over time in Britain and Germany. Contrary to expectations they find that changing job insecurity does not explain the modest fall in job satisfaction in either country, but intensification of work effort and declining task discretion can explain part of the decline in job satisfaction in Britain. In this paper we focus on regional differences in job satisfaction within a single country, which to our knowledge has not been analysed before. To the extent that there are regional differences in earnings we might expect this to be reflected in reported job satisfaction. However, this may be moderated by the fact that lower earnings go hand in hand with higher levels of unemployment and inactivity, so that being in work may be regarded more favourably in more economically depressed regions.

We make use of the British Household Panel Survey (BHPS) which asks individuals all things considered, how satisfied or dissatisfied they are with their present job 
overall on a scale of 1 to 7 , where 1 represents completely dissatisfied, 4 neither satisfied nor dissatisfied and 7 completely satisfied. Similar questions using the same scale were asked about total pay (including any overtime or bonuses), job security, hours of work and the actual work itself. Recent boosts to the BHPS in Scotland and Wales have increased the sample size, which allows for more detailed regional comparisons. Further, waves $6-10$ contained questions on overall life satisfaction which allows for comparisons in each region between life satisfaction of the employed, unemployed and inactive and job satisfaction.

Earlier studies have shown that low-paid workers have job satisfaction which is as high, if not higher, than higher paid workers, though in part this may be the result of compositional effects (Leontaridi, Sloane and Jones 2004.) This paper extends the analysis to regions with differing concentrations of high and low paid workers and enables us to control for both industry and occupational groups. 


\section{MODEL SPECIFICATION AND ESTIMATION}

Our starting point is that job satisfaction is a reasonable proxy for the utility of work. As Hamermesh (2001) suggests,

"A potentially useful view is that job satisfaction is the resultant of the worker's weighing in his/her mind of all the job’s aspects. It can be viewed as a single metric that allows a worker to compare the current job to other labour market opportunities."

Of course, we cannot be certain that individual workers will use the 1 to 7 ranking scale in exactly the same way, but empirical regularities can be observed using such job satisfaction measures. Thus, Hamermesh (1977), Freeman (1978), Akerlof, Rose and Yellen (1988) and Clark (2001) have all found that recorded job satisfaction is a strong predictor of quit behaviour, while Mangione and Quinn (1975) and Clegg (1983) found that there was a negative correlation between job satisfaction and both worker absence and productivity. Job satisfaction can be considered to be a type of sub-utility function u within an overall utility function, v, representing overall life satisfaction.

Thus, $\mathrm{v}=\mathrm{v} \quad\{\mathrm{u}(\mathrm{h}, \mathrm{i}, \mathrm{j}) \mu\}$

Where $\mathrm{u}$ is the utility from work and $\mu$ the utility obtained from other sources or non-work spheres of activity. As work is an important component of life in general

\footnotetext{
${ }^{2}$ As Kristensen and Westergaard-Nielsen (2004) point out, if job satisfaction is used on the right-hand side to explain variables such as quit behaviour and simply depended on personal sentiments and the standard observed variables such as age and education, no new or additional information would be contained in measures of job satisfaction themselves which would then be endogenous. However, this variable is likely to capture unobservables such as work organisation, physical work conditions and the workers perception of the quality of the job match which allow for exogenous variation.
} 
we would expect there to be a positive association between utility from work and overall life satisfaction. The utility from work then takes the form,

$\mathrm{u}=\mathrm{u}(\mathrm{y}, \mathrm{h}, \mathrm{i}, \mathrm{j})$

where y equals wage income, h represents hours of work and i and $\mathrm{j}$ are vectors of individual and job specific characteristics, respectively.

A prominent view in the psychology literature is that happiness, in part, depends on relative income or what others earn. In the economics literature Easterlin (1974) first put forward the hypothesis that overall well being depended on relative rather than absolute income in the context of inter-country comparisons and within-country time series. Rees (1993) also suggested that there was an inverse relationship between a worker's satisfaction and the pay of other workers. Baxter (1973 and 1993) formalised this in the concept of relative deprivation. That is, as a worker's level of earnings falls relative to that of others, the individual will feel relatively deprived and happiness will decline. To incorporate this idea we extend the utility function by including an additional variable $\mathrm{y}^{*}$ to proxy an individual's reference income. Thus, $\mathrm{u}=\mathrm{u}\left(\mathrm{y}, \mathrm{y}^{*}, \mathrm{~h}, \mathrm{i}, \mathrm{j}\right)$

We do not directly observe $\mathrm{y}^{*}$ but the psychology literature suggests that such comparisons tend to be narrowly drawn. Thus, Major and Forcey (1985) found that individuals preferred to make comparisons within the same sex and job rather than across these dimensions. ${ }^{3}$ Our reference income is obtained by estimating a wage equation and then using the results to estimate a predicted wage for each individual based on their personal and job characteristics. There are potential econometric

\footnotetext{
${ }^{3}$ Frank (1985) showed that wage distributions within firms were much more compressed than would be expected if relative income were unimportant. Further, the incidence of piece-work pay was much lower and the frequency of strikes much higher than if this were not the case.
} 
problems in making comparison income the residual in a human capital regression. Therefore, we derive our comparison pay from the Labour Force Survey (LFS) corresponding to the date of interview ${ }^{4}$. Thus, if actual pay is below the pay predicted from the LFS we would expect this to reduce job satisfaction.

A further consideration is that job satisfaction and wages may be endogenous. For example, wages and job satisfaction could be simultaneously determined were wages to compensate for the degree of risk in a job which, in turn, could lower job satisfaction if an individual were risk averse. Or, suppose more satisfied workers tend to increase the degree of work effort and this, in turn, raises pay, then again the two variables will be endogenous. There are standard ways of dealing with this problem, but finding appropriate exclusion restrictions can be problematical. Lydon and Chevalier (2002) made a rare attempt to deal with the problem by using characteristics of a respondent's partner or spouse as instruments in a sample of graduates and this produced significantly higher own wage effects in their job satisfaction equation than when wages were treated as exogenous. However, this result is obtained at the cost of restricting the sample to married individuals or those with partners and we do not attempt to deal with this potential problem here. Failing to deal with this problem may be less crucial in our case, given that our main concern is with regional differences in job satisfaction.

One advantage of using the BHPS is that one can make use of the panel element to control for individual heterogeneity. The unobserved component can be treated as either a random variable or as a fixed effect, when it is treated as a parameter to be

\footnotetext{
${ }^{4}$ Because we have observations of pay in different years we deflate the pay variable by the retail price index so that we are always considering real pay.
} 
estimated for each cross section observation. Choosing between these two alternatives is not straightforward, but since the BHPS draws individuals randomly from a large population the random effects approach is an appropriate specification and avoids a loss of degrees of freedom that would result from using a fixed effects model. Further, when using fixed effects it is not possible to distinguish between the effects of time-constant observables and those of the time-constant unobservables, so that individual factors such as gender cannot be included as independent regressors. Such variables are important in the context of our study and hence we utilise a random effects ordered probit model. 


\section{SOME DESCRIPTIVES}

According to wave 11 of the BHPS in 2002 job satisfaction was higher in Wales than in any other part of Britain. Looking at the descriptive statistics (table 2) features tending to raise job satisfaction in Wales on the basis of earlier studies are a high female/male employment ratio, high job tenure and age, a low proportion with university degrees, high public sector employment and low travel to work times. Features tending to lower job satisfaction in Wales relative to elsewhere are low wages, high hours of work, a high proportion of home-owners ${ }^{5}$, less extensive promotion prospects and high trade union membership.

\section{Table One about here.}

In every region there has been some decline in recorded levels of job satisfaction compared to wave 1 (1991), though a degree of caution is required interpreting these results due to the small sample size in Scotland and particularly Wales prior to the boosts to the BHPS in these two regions (figure one). Further, there has been some increase in recorded job satisfaction over the last three waves. It should also be noted that in contrast to the trend in overall job satisfaction, there has been an upward trend between waves 1 and 11 in the recorded levels of satisfaction with pay (figure two), with Wales having the highest recorded level at the end of the period despite the lower mean level of pay there.

\footnotetext{
${ }^{5}$ A number of studies (e.g. Clark and Oswald, 1996, and Sloane and Williams, 2000 have found a relationship between home ownership (or more particularly having a mortgage) and job satisfaction. The need to pay for the costs of a mortgage may increase the need to earn more and thereby increase the intensity of work and reduce job satisfaction, but there are other possibilities. Thus Clark (1996), who finds renters are more satisfied at work than home owners, attributes this to renting making it easier to change jobs and, therefore, get a better job match.
} 
One reason for variation in job satisfaction across regions is that the variable may depend on possible alternatives such as unemployment and inactivity - as Kristensen and Westergaard-Nielsen (2004) point out one would expect individuals with good outside alternative job opportunities to be less satisfied than individuals with none. They find that average job satisfaction increases with the unemployment rate.

In terms of overall life satisfaction, data for which are available up to wave 10, Wales also has the highest recorded level at the end of the period, with the level being stable between waves 8 and 10 when there were declines in the other regions (figure three). When the sample is divided into employed, unemployed and inactive (figure five) life satisfaction is clearly higher for the employed group and lowest for the unemployed group in each of the regions. The sample was further divided into those expressing low job satisfaction (1 and 2), medium job satisfaction (3, 4 and 5) and high job satisfaction (6 and 7), (figure three). Overall life satisfaction was clearly highest for those with high job satisfaction and lowest for those with low job satisfaction, the same being true for all regions.

The finding of high overall life satisfaction in Wales is not quite matched by the inactive group which record figures not very different from those in other regions. This suggests that in the regression analysis we should control for sample selection, by using the Heckman two step procedure. However, inserting the inverse Mills ratio into an ordered probit equation is not a standard procedure and could itself insert a bias into our results. Therefore, in our reported results we merely attempt to insert additional explanatory variables into our estimating equations to pick up such effects. 


\section{2. $\quad$ Results}

Experimentation with different regions led to the conclusion that splitting Great Britain into four regions would make for the neatest comparisons, as division into smaller regions reduces sample size. Thus, our regions are London and the SouthEast, the Rest of England, Scotland and Wales. We then combine waves 9, 10 and 11, the years when the boosts are available for Scotland and Wales. This enables us to apply a random effects ordered probit model to correct for unobservables, in which observations in separate years for the same individuals are treated as separate observations. We utilise an unbalanced panel as restricting the analysis to a balanced panel leads to a substantial decline in sample size since less than half of the individuals in the unbalanced panel answer the job satisfaction question in the three waves being considered. We estimate a balanced panel for the national sample and find that the coefficients are very similar to those obtained using the unbalanced panel estimation, but generally have larger standard errors.

Examining first the results for the whole sample, (Table 2), it is clear that Wales is different. With Wales as the omitted category overall job satisfaction is significantly lower in the other three regions. We tested whether this is due to being Welsh or living in Wales by estimating the model including a dummy variable indicating whether the individual was born in Wales or not. The variable was found to be insignificant at conventional levels suggesting that it is living in Wales which is important. The wave dummies are consistent with a decline in job satisfaction over this period. In general the results are in line with those of earlier studies using the BHPS. Women express themselves as more satisfied at work than men ${ }^{6}$ and the level

${ }^{6}$ These results are not reported here, but are available on request. 
of pay does not significantly raise the level of satisfaction of women, unlike the case with men. ${ }^{7}$ Indeed, when interaction terms between gender and the absolute pay variable were included in the overall regression the coefficient was positive and significant $(\mathrm{t}=4.17)$, indicating that earnings have a significantly greater impact on the job satisfaction of men than of women ${ }^{8}$.

There is a problem of multi-collinearity, however, when we include comparison pay, age and education in the same equations, as comparison pay becomes insignificant in this case. If education and age are omitted comparison pay becomes significant both for men and for women. Hence we report equations with and without the comparison pay variable. The absolute pay variable is gross usual monthly pay converted to an hourly rate and hours are usual hours. As expected, hours of work are negative and significant so that, ceteris paribus workers prefer shorter hours. We also include the pattern of hours. It appears that workers dislike most working in the evenings or nights, though on its own, working at night is insignificant at conventional levels. There are, however, some gender differences. Women, but not men, have a preference for working in the mornings only, but do not have significantly lower job satisfaction from working evenings or nights, while men appear to dislike split shifts and rotating shifts.

Similarly, while men employed in the public sector have lower satisfaction than those employed in the private sector, the sign on public sector employment is positive for women. For both sexes job satisfaction is higher for part-time workers

\footnotetext{
7 Sousa-Poza and Sousa-Poza (2003) show using the first 10 waves of the BHPS that women's satisfaction declined substantially in the 1990's, whereas men's job satisfaction remained fairly constant. This resulted in a halving of the gender job satisfaction differential. Nevertheless, we find that the difference is still significant.

${ }^{8}$ However in the case of Wales we find this term is insignificantly different from zero.
} 
than full-time workers. In terms of other job characteristics the availability of promotion opportunities, together with incremental pay systems, employment in small establishments with less than 100 employees and shorter journey to work times significantly raise job satisfaction. As for personal characteristics we find that satisfaction is $\mathrm{U}$ shaped in age and tenure, increases with good health and declines with higher levels of education and home ownership. There is also a significant negative relationship with trade union membership which may reflect omitted variables such as the quality of industrial relations or alternatively that dissatisfied workers are more likely to join unions. ${ }^{9}$ To see whether an individual's recent work history plays some part in explaining differences in satisfaction, we include two variables, one of which measures the number of weeks in which an individual was not working in the previous year and the other which is a dummy for whether or not the individual voluntarily quit his or her last job. Both of these turn out to be highly significant with expected signs.

When the sample is split by region the most striking feature for Wales (Table 2), is that comparative pay is never a significant determinant of job satisfaction for either men or women and for the latter usual pay is never significant either. For women the voluntary quits and inactivity variables are insignificant at conventional levels. This story is, in the main, replicated for most variables, in the other regions, save for the fact that the comparative wage is significant for both genders in the Rest of England for men (at the 10\% level) in London and the South-East and (also at the

\footnotetext{
${ }^{9}$ Bender and Sloane (1998), using the Social and Economic Life Initiative dataset, found that when a variable measuring the quality of industrial relations was included in a job satisfaction equation the negative sign on trade union membership disappeared. Consistent with this hypothesis Drinkwater and Ingram (2003) found using the Social Attitudes Survey that workers in Wales were significantly more likely to report good industrial relations and workplace harmony than workers in other regions.
} 
$10 \%$ level) for all workers in Scotland. This variable is also significant for women, as well as men, in Scotland and the Rest of England, where the inactivity variable is also significant for women at the $10 \%$ level.

When the regressions were run with education excluded real predicted pay, always significant at the national level, was also significant for women in Scotland, and for both men and women in London and the South-East and the Rest of England. However, it was insignificant in Wales. This points to the fact that pay appears to have a less important effect on job satisfaction in Wales than it does in the rest of Britain. $^{10}$

We also include a model for Wales with a dummy variable included for areas with Objective One status. The reason for this is that West Wales and the Valleys receive substantial support from the European Social Fund as GDP in these areas is less than $75 \%$ of the European Union average. If higher job satisfaction in Wales is a consequence of higher inactivity in Wales, then we would also expect that job satisfaction would be higher in West Wales and the Valleys for the same reason. By way of illustration according to the Welsh Local Labour Force Survey 2001-2002 the inactivity rate (working age) was $28.6 \%$ for the Objective One area compared to 23.9\% for the Objective Three area (the rest of Wales) and according to NES data the gross weekly wage in 2002 in the Objective One area was £384.88 compared to $£ 418.36$ in the Objective Three area. There is a slight problem in defining our dummy variable as the BHPS combines Torfaen (in the Objective One area) with the

\footnotetext{
${ }^{10}$ Oswald (1997) found that overall happiness was determined much more by whether the individual had a job than by the level of income. The worst thing about losing a job was not the drop in takehome pay but the non-pecuniary distress which it caused. Mental distress was twice as high among the unemployed as among the employed.
} 
neighbouring Monmouthshire (in the Objective 3 area), so it is necessary to drop these Unitary Authorities from our analysis with the Objective One dummy included. This results in a loss of $8.66 \%$ of the observations from the Welsh sample, which is sufficiently small that it should not invalidate the exercise.

The sign on the Objective One coefficient is always positive and significant at the $10 \%$ level for the whole sample and for women. However, regional unemployment and inactivity rates were insignificant when included in the jobs satisfaction equations and did not affect the significance of the regional dummies. ${ }^{11}$ Thus, it seems unlikely that low activity rates for Wales explain the high levels of job satisfaction there. This is reinforced by the fact that job satisfaction is significantly higher in Wales than elsewhere after controlling for number of weeks not employed in the last year. ${ }^{12}$

It should also be bourne in mind that it is the younger and more educated that tend to migrate from Wales and outward migration is substantial. In April 2001 1.24\% of those resident in England were born in Wales (Drinkwater and Blackaby, 2004). Thus, some of the more dissatisfied workers in Wales in the past, may now be working outside of Wales. However, when a dummy variable for born in Wales was included in a job satisfaction equation for England it turned out to be insignificant. Thus, the Welsh in England are similar to those born in England. However, we

\footnotetext{
${ }^{11}$ We also change our omitted group in the national regressions from Wales to the objective one region in Wales. Thus we included the objective three in Wales region as another region. We find that London and the South East, the rest of England and Scotland are significantly different from the Wales objective one region but the Wales objective three region is not.

${ }^{12}$ We also included 16 local authority dummies to see if there were significant differences in job satisfaction at this level of dis-aggregation. Four local authority areas had significantly higher than elsewhere (at the $10 \%$ level or higher), all of them in the Objective One area.
} 
cannot identify if whether this is a consequence of location or movement into a better job.

We also attempted a decomposition based on the predictions of the ordered probit model using the Welsh coefficients with the characteristics means from the other regions. If those in Wales had the same average characteristics as those in other regions mean satisfaction would rise slightly for all workers and women more substantially in comparison to London and the South East. Therefore, it does not appear that the personal characteristics in our model are driving the higher job satisfaction found in Wales. ${ }^{13}$

\footnotetext{
${ }^{13}$ We also experimented with OLS and found that the major difference was in the intercept term, suggesting that omitted variables were driving the higher job satisfaction found in Wales.
} 


\section{CONCLUSIONS}

The aim of this paper was to explain why job satisfaction should be significantly higher in Wales than in the rest of Britain, despite the lower GDP per head employment and earnings in that region. There are a number of possible explanations for these findings. First, the higher levels of inactivity and unemployment may lead to the in work state being regarded more favourably than where job openings are more plentiful.

We find that those individuals who had a period out of work in the last year and those who quit their last job voluntarily had higher levels of satisfaction in their current job, but this is not sufficient to remove the significantly higher job satisfaction in Wales. Further, job satisfaction is just as high in the economically depressed Objective One area of Wales as in the rest of Wales and, in the case of women, higher.

Another possibility is that the climate of industrial relations, as perceived by workers, is better in Wales than elsewhere and there is evidence for this from another study. (Drinkwater and Ingram 2003). This is important as Wales has relatively high union density, a factor which generally lowers job satisfaction. A third possibility is that workers in Wales are less concerned about their level of pay than workers elsewhere. Hourly wages are only significant at the $10 \%$ level for workers in Wales, as opposed to the $1 \%$ level for Britain as a whole and relative wages are insignificant, and for women in Wales hourly wages are insignificant too (though this is also the case elsewhere). 
A final possibility is that the most dissatisfied workers in Wales tend to move out of the region and increase recorded job satisfaction for those who remain there, but because personal characteristics of those in Wales are not more favourable to high job satisfaction than in other regions suggests that this is not a major cause of the difference.

It may be that socio-psychological factors or cultural factors which we cannot control for are responsible for some of these differences. There is, for example, a particular Welsh culture and identity, reinforced by a separate language, which may play some part in increasing job satisfaction in addition to the factors which we have referred to in the paper. Whatever the cause there may be some labour market advantages for firms choosing to locate in Wales, given the combination of relatively higher job satisfaction and relatively low wages. 


\section{TABLE 1}

\begin{tabular}{|l|l|l|l|}
\hline \multicolumn{2}{|l|}{ JOB SATISFACTION IN 2002} & WOMEN & ALL WORKERS \\
\hline & MEN & & \\
\hline London and the South East & 5.23 & 5.49 & 5.37 \\
\hline Rest of England & 5.22 & 5.49 & 5.36 \\
\hline Scotland & 5.15 & 5.46 & 5.32 \\
\hline Wales & 5.40 & 5.67 & 5.54 \\
\hline Great Britain & 5.23 & 5.49 & 5.37 \\
\hline
\end{tabular}


Table 2A: Descriptive Statistics: Males and Females

\section{Wales}

(including (excluding

objective 1 objective 1

National

Job Satisfaction

Gender

Log usual real gross hourly

wage

Log usual hours

Job Tenure

Tenure Squared

Age

Age square

University degree

Vocational qualifications

A - levels plus

$\mathrm{O}$ - levels plus

Commercial or

Apprentice

Married

Mortgage house

Paid outright

house

Promotion

Opportunities

Public Sector

Works mornings

only

Works evenings

or nights

Variable shift

patterns

Fair health

Good health

Excellent health

Trade union

member

Temporary Job

Incremental pay

Part Time

Travel Time

Size 25 - 99

Size 100 - 499

Size 500 plus

Utilities

Construction

Retail

Catering

Transport

Financial

\subsection{2}

0.47

\subsection{5}

3.46

52.84

7327.00

37.24

1509.22

0.17

0.31

0.15

0.20

0.07

0.56

0.66

0.12

0.52

0.32

0.05

0.05

0.13

0.23

0.46

0.25

0.32

0.06

0.47

0.80

23.32

0.26

0.23

0.18

0.02

0.03

0.08

0.08

0.03

0.19

variable)

5.45

0.48

variable)

5.45

0.48

1.68

3.46

61.51

9104.82

37.68

1541.17

0.14

0.30

0.15

0.19

0.08

0.60

0.71

0.14

0.51

0.35

0.06

0.05

0.17

0.21

0.43

0.28

0.40

0.06

0.45

0.79

20.23

0.25

0.23

0.18

0.02

0.04

0.09

0.10

0.04

0.19

$\begin{array}{rr}1.69 & 1.75 \\ 3.46 & 3.46 \\ 62.61 & 58.75 \\ 9476.65 & 8573.86 \\ 37.92 & 36.75 \\ 1558.39 & 1467.66\end{array}$

0.13

0.20

0.30

0.28

0.15

0.20

0.17

0.19

0.08

0.62

0.04

0.55

0.71

0.66

0.15

0.08

0.51

0.35

0.55

0.38

0.05

0.04

$0.05 \quad 0.05$

0.17

0.21

0.43

0.28

0.40

0.05

0.44

0.79

20.31

0.25

0.23

0.18

0.02

0.05

0.09

0.09

0.04

0.18
London and

South East Rest of England

$\begin{array}{ll}5.26 & 5.31 \\ 0.46 & 0.48\end{array}$

1.90

1.70

$3.48 \quad 3.46$

$42.21 \quad 51.67$

$5075.30 \quad 7054.03$

37.71

1556.90

0.22

0.32

0.13

0.18

0.07

0.52

0.61

0.13

0.53

0.32

0.05

0.03

0.09

0.23

0.48

0.23

0.24

0.06

0.47

0.82

29.32

0.26

0.22

0.19

0.01

0.02

0.07

0.06

0.03

0.18
7054.03

37.01

1488.34

0.15

0.32

0.14

0.21

0.08

0.57

0.67

0.12

0.50

0.28

0.05

0.05

0.13

0.24

0.46

0.22

0.31

0.05

0.44

0.79

21.50

0.25

0.25

0.17

0.01

0.04

0.09

0.09

0.03

0.20 


\begin{tabular}{|c|c|c|c|c|c|c|}
\hline Other business & 0.06 & 0.05 & 0.05 & 0.06 & 0.06 & 0.07 \\
\hline Public & 0.13 & 0.09 & 0.10 & 0.12 & 0.19 & 0.12 \\
\hline Other & 0.36 & 0.37 & 0.37 & 0.41 & 0.37 & 0.33 \\
\hline Professional & 0.10 & 0.10 & 0.10 & 0.10 & 0.12 & 0.09 \\
\hline \multicolumn{7}{|l|}{ Associate } \\
\hline Professional & 0.12 & 0.10 & 0.10 & 0.14 & 0.15 & 0.11 \\
\hline Clerical & 0.19 & 0.17 & 0.17 & 0.18 & 0.20 & 0.19 \\
\hline Services & 0.21 & 0.23 & 0.23 & 0.21 & 0.18 & 0.22 \\
\hline Sales & 0.08 & 0.07 & 0.07 & 0.08 & 0.07 & 0.08 \\
\hline \multicolumn{7}{|l|}{ Operative and } \\
\hline Assembly & 0.09 & 0.12 & 0.12 & 0.07 & 0.05 & 0.11 \\
\hline Other & 0.08 & 0.08 & 0.08 & 0.09 & 0.06 & 0.08 \\
\hline Voluntarily Quit & 0.09 & 0.07 & 0.07 & 0.10 & 0.10 & 0.09 \\
\hline \multicolumn{7}{|l|}{$\begin{array}{l}\text { Number of weeks } \\
\text { not employed in }\end{array}$} \\
\hline the last year & 3.06 & 3.10 & 2.99 & 3.58 & 3.21 & 2.75 \\
\hline Wave10 & 0.34 & 0.34 & 0.34 & 0.34 & 0.33 & 0.34 \\
\hline Wave11 & 0.32 & 0.32 & 0.32 & 0.32 & 0.32 & 0.32 \\
\hline Rest of England & 0.42 & & & & & \\
\hline London and & & & & & & \\
\hline South East & 0.21 & & & & & \\
\hline Scotland & 0.21 & & & & & \\
\hline $\begin{array}{l}\text { Objective one } \\
\text { area }\end{array}$ & & & & & & \\
\hline
\end{tabular}


Table 2B: Descriptive Statistics: Males Only

Wales Wales

(including (Excluding

objective 1 objective 1

National variable) variable)

Job

Satisfaction

5.34

gross hourly

wage

Log usual

hours

Job Tenure

Tenure

Squared

Age

Age square

University

degree

Vocational

qualifications

A - levels plus

$\mathrm{O}$ - levels plus

Commercial or

Apprentice

Married

Mortgage

house

Paid outright

house

Promotion

Opportunities

Public Sector

Works

mornings only

Works

evenings or

nights

Variable shift

patterns

Fair health

Good health

Excellent

health

Trade union

member

Temporary Job

Incremental

pay

Part Time

Travel Time

Size 25 - 99

Size 100 - 499

Size 500 plus

sic2

sic3
5.20

1.88

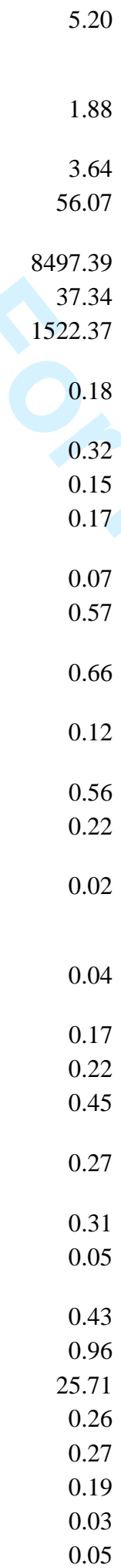

1.82

3.64

66.82

10848.60

37.36

1520.52

$$
0.15
$$

0.33

0.14

0.17

$$
0.07
$$

0.60

0.72

11165.59

37.71

1547.11

0.15

0.33

0.14

0.18

0.07

0.61

0.72

0.14

0.14

0.55

0.25

0.54

0.24

0.03

0.03

0.04

0.03

0.22

0.21

0.22

0.20

0.43

0.43

0.29

0.29

0.41

0.05

0.41

0.96

22.52

0.26

0.28

0.18

0.03

0.08

0.42

0.04

0.41

0.96

22.97

0.28

0.19

0.03
5.35

London and

Scotland South East Rest of England

$$
5.17
$$$$
5.14
$$$$
5.18
$$

1.84

2.05

1.83

3.65

53.79

64.88

3.64
43.28

7897.62

37.05

36.91

5598.66

38.08

1496.66

(20

1588.19

0.16

0.29

0.19

0.18

0.04

0.56

0.67

0.08

0.58

0.28

0.02

0.24

0.32

0.34

0.14

0.18

0.16

0.09

0.57

0.54

0.61

0.66

0.14

0.12

0.57

0.54

0.17

0.02

0.02

0.04

0.18

0.17

0.21

0.44

0.24

0.45

0.45

0.29

0.27

0.24

0.33

0.23

0.31

0.05

0.05

0.04

0.49

0.43

0.40

0.94

0.96

0.96

24.50

33.34

23.58

0.26

0.24

0.26

0.24

0.26

0.29

0.23

0.20

0.16

0.04

0.02

0.02

0.03

0.03

0.06 


\begin{tabular}{|c|c|c|c|c|c|c|}
\hline sic4 & 0.14 & 0.15 & 0.16 & 0.12 & 0.12 & 0.15 \\
\hline sic5 & 0.11 & 0.14 & 0.13 & 0.10 & 0.07 & 0.13 \\
\hline sic6 & 0.06 & 0.08 & 0.08 & 0.07 & 0.05 & 0.06 \\
\hline sic7 & 0.15 & 0.12 & 0.12 & 0.14 & 0.15 & 0.16 \\
\hline sic8 & 0.09 & 0.08 & 0.07 & 0.09 & 0.10 & 0.10 \\
\hline sic9 & 0.14 & 0.09 & 0.09 & 0.13 & 0.21 & 0.13 \\
\hline sic10 & 0.22 & 0.23 & 0.23 & 0.27 & 0.25 & 0.19 \\
\hline $\begin{array}{l}\text { Professional } \\
\text { Associate }\end{array}$ & 0.10 & 0.10 & 0.11 & 0.10 & 0.12 & 0.09 \\
\hline Professional & 0.11 & 0.07 & 0.07 & 0.11 & 0.15 & 0.10 \\
\hline Clerical & 0.11 & 0.09 & 0.09 & 0.13 & 0.10 & 0.10 \\
\hline Services & 0.25 & 0.27 & 0.27 & 0.26 & 0.21 & 0.25 \\
\hline Sales & 0.04 & 0.03 & 0.03 & 0.04 & 0.05 & 0.04 \\
\hline $\begin{array}{l}\text { Operative and } \\
\text { Assembly }\end{array}$ & 0.15 & 0.20 & 0.20 & 0.12 & 0.09 & 0.18 \\
\hline $\begin{array}{l}\text { Other } \\
\text { Voluntarily }\end{array}$ & 0.07 & 0.09 & 0.08 & 0.08 & 0.06 & 0.08 \\
\hline Quit & 0.10 & 0.07 & 0.08 & 0.09 & 0.12 & 0.09 \\
\hline $\begin{array}{l}\text { Number of } \\
\text { weeks not } \\
\text { employed in }\end{array}$ & & & & & & \\
\hline the last year & 2.41 & 2.72 & 2.59 & 2.79 & 2.57 & 2.08 \\
\hline Wave10 & 0.34 & 0.34 & 0.34 & 0.34 & 0.33 & 0.34 \\
\hline Wave11 & 0.32 & 0.31 & 0.31 & 0.32 & 0.33 & 0.31 \\
\hline $\begin{array}{l}\text { Rest of } \\
\text { England }\end{array}$ & 0.43 & & & & & \\
\hline $\begin{array}{l}\text { London and } \\
\text { South East }\end{array}$ & 0.21 & & & & & \\
\hline Scotland & 0.20 & & & & & \\
\hline $\begin{array}{l}\text { Objective one } \\
\text { area }\end{array}$ & & 0.61 & & & & \\
\hline
\end{tabular}


Table 2C: Descriptive Statistics: Females Only

$\begin{array}{cc}\text { Wales } & \text { Wales } \\ \text { (including } & \text { (Excluding }\end{array}$

objective 1 objective 1

National variable) variable) Scotland South East Rest of England

Job Satisfaction

5.43

5.54

5.54

5.41

5.37

5.43

Log usual rea

gross hourly

wage

1.64

1.57

1.57

1.67

1.78

1.58

usua

hours

3.31

3.28

3.29

3.34

3.34

3.28

Tenure Squared

49.98

56.58

57.75

53.75

41.30

49.73

Age

6291.87

7481.82

7888.88

6871.15

4628.58

6280.05

37.15

38.11

36.61

37.39

36.96

Age square

1497.59

1560.39

1569.00

1449.48

1530.19

1480.71

University

degree

0.16

0.13

0.12

0.20

0.20

0.14

qualifications

0.29

0.28

0.28

0.28

0.32

0.29

A - levels plus

0.16

0.15

0.13

0.14

0.22

0.22

0.22

0.20

0.20

0.24

Commercial or

0.07

0.09

0.09

0.05

0.07

0.07

Married

0.56

0.61

0.62

0.54

0.50

0.57

Mortgage

house

0.66

0.70

0.70

0.65

0.61

0.69

house

0.11

0.15

0.15

0.08

0.13

0.11

Promotion

Opportunities

0.49

0.47

0.48

0.53

0.50

0.47

Works

mornings only

0.41

0.45

0.44

0.46

0.39

0.38

0.07

0.08

0.07

0.06

0.07

0.07

evenings or

nights

0.05

0.07

0.07

0.05

0.04

0.06

ariable shift

patterns

0.10

0.12

0.12

0.14

0.07

0.09

0.23

0.22

0.21

0.22

0.24

0.25

Good health

0.44

0.44

0.45

0.50

0.47

Excellent

health

0.23

0.27

0.27

0.27

0.20

0.20

Trade union

member

0.32

0.38

0.38

0.37

0.25

0.31

Temporary Job

0.06

0.06

0.06

0.07

0.06

pay

0.50

0.48

0.47

0.58

0.51

0.47

Part Time

0.66

0.64

0.64

0.68

0.70

0.63

18.10

17.80

21.93

25.89

19.60

Size 25 - 99

0.26

0.25

0.25

0.28

0.25

0.18

0.18

0.27

0.19

0.20

Size 500 plus

0.17

0.16

0.17

0.18

0.17

0.01

0.01

0.01

0.01

0.01

sic3

0.01

0.01

0.01

0.01

0.02

sic4

0.03

0.04

0.03

0.03

0.04

sic5

0.05

0.06

0.06

0.05

0.05

0.06 


\begin{tabular}{|c|c|c|c|c|c|c|}
\hline sic6 & 0.01 & 0.01 & 0.01 & 0.01 & 0.01 & 0.01 \\
\hline sic7 & 0.23 & 0.24 & 0.25 & 0.22 & 0.21 & 0.24 \\
\hline sic8 & 0.04 & 0.03 & 0.03 & 0.04 & 0.03 & 0.04 \\
\hline $\operatorname{sic} 9$ & 0.13 & 0.10 & 0.10 & 0.12 & 0.18 & 0.12 \\
\hline sic10 & 0.48 & 0.50 & 0.50 & 0.52 & 0.47 & 0.45 \\
\hline $\begin{array}{l}\text { Professional } \\
\text { Associate }\end{array}$ & 0.10 & 0.09 & 0.09 & 0.10 & 0.12 & 0.09 \\
\hline Professional & 0.13 & 0.13 & 0.13 & 0.16 & 0.15 & 0.11 \\
\hline Clerical & 0.26 & 0.25 & 0.25 & 0.22 & 0.29 & 0.28 \\
\hline Services & 0.18 & 0.20 & 0.20 & 0.17 & 0.16 & 0.18 \\
\hline Sales & 0.11 & 0.12 & 0.11 & 0.12 & 0.09 & 0.11 \\
\hline $\begin{array}{l}\text { Operative and } \\
\text { Assembly }\end{array}$ & 0.04 & 0.05 & 0.05 & 0.04 & 0.02 & 0.04 \\
\hline $\begin{array}{l}\text { Other } \\
\text { Voluntarily }\end{array}$ & 0.08 & 0.07 & 0.07 & 0.09 & 0.06 & 0.08 \\
\hline $\begin{array}{l}\text { Quit } \\
\text { Number of } \\
\text { weeks not } \\
\text { employed in }\end{array}$ & 0.09 & 0.07 & 0.07 & 0.10 & 0.09 & 0.09 \\
\hline the last year & 3.65 & 3.46 & 3.36 & 4.22 & 3.76 & 3.38 \\
\hline Wave10 & 0.33 & 0.33 & 0.33 & 0.33 & 0.34 & 0.34 \\
\hline Wave11 & 0.32 & 0.32 & 0.32 & 0.33 & 0.31 & 0.32 \\
\hline $\begin{array}{l}\text { Rest of } \\
\text { England }\end{array}$ & 0.41 & & & & & \\
\hline $\begin{array}{l}\text { London and } \\
\text { South East }\end{array}$ & 0.22 & & & & & \\
\hline Scotland & 0.22 & & & & & \\
\hline $\begin{array}{l}\text { Objective one } \\
\text { area }\end{array}$ & & 0.61 & & & & \\
\hline
\end{tabular}


Table 3A: OVERALL JOB SATISFACTION - Males and Females

National

\begin{tabular}{|c|c|c|c|c|c|c|}
\hline & & & 1 variable & & objective & able \\
\hline & (1) & (2) & (3) & (4) & & (6) \\
\hline Gender & $\begin{array}{l}-0.218^{* * *} \\
(6.785)\end{array}$ & $\begin{array}{l}-0.205^{* * *} \\
(6.393)\end{array}$ & $\begin{array}{l}-0.204 * * \\
(2.401)\end{array}$ & $\begin{array}{l}-0.221^{* * *} \\
(2.584)\end{array}$ & $\begin{array}{l}-0.158^{* *} \\
(1.965)\end{array}$ & $\begin{array}{l}-0.175^{* *} \\
(2.169)\end{array}$ \\
\hline $\begin{array}{l}\text { Log usual real } \\
\text { gross hourly } \\
\text { wage }\end{array}$ & $\begin{array}{l}0.180 * * * \\
(5.263)\end{array}$ & $\begin{array}{l}0.120 * * * \\
(3.661)\end{array}$ & $\begin{array}{l}0.181^{*} \\
(1.912)\end{array}$ & $\begin{array}{l}0.125 \\
(1.355)\end{array}$ & $\begin{array}{l}0.154^{*} \\
(1.717)\end{array}$ & $\begin{array}{l}0.111 \\
(1.272)\end{array}$ \\
\hline Log usual hours & $\begin{array}{l}-0.214^{* * *} \\
(4.195)\end{array}$ & $\begin{array}{l}-0.221^{* * *} \\
(4.341)\end{array}$ & $\begin{array}{l}-0.112 \\
(0.878)\end{array}$ & $\begin{array}{l}-0.116 \\
(0.904)\end{array}$ & $\begin{array}{l}-0.073 \\
(0.597)\end{array}$ & $\begin{array}{l}-0.077 \\
(0.632)\end{array}$ \\
\hline Job Tenure & $\begin{array}{l}-0.002^{* * *} \\
(4.534)\end{array}$ & $\begin{array}{l}-0.002^{* * *} \\
(3.185)\end{array}$ & $\begin{array}{l}-0.002 \\
(1.559)\end{array}$ & $\begin{array}{l}-0.002 \\
(1.156)\end{array}$ & $\begin{array}{l}-0.003^{* *} \\
(2.327)\end{array}$ & $\begin{array}{l}-0.002 * * \\
(1.970)\end{array}$ \\
\hline Tenure Squared & $\begin{array}{l}0.000 * * * \\
(3.121)\end{array}$ & $\begin{array}{l}0.000 * * * \\
(3.071)\end{array}$ & $\begin{array}{l}0.000 \\
(1.093)\end{array}$ & $\begin{array}{l}0.000 \\
(1.293)\end{array}$ & $\begin{array}{l}0.000^{*} \\
(1.939)\end{array}$ & $\begin{array}{l}0.000^{* *} \\
(2.179)\end{array}$ \\
\hline Age & $\begin{array}{l}-0.023^{* *} \\
(2.550)\end{array}$ & & $\begin{array}{l}-0.001 \\
(0.060)\end{array}$ & & $\begin{array}{l}0.001 \\
(0.050)\end{array}$ & \\
\hline Age square & $\begin{array}{l}0.000 * * * \\
(3.306)\end{array}$ & & $\begin{array}{l}0.000 \\
(0.622)\end{array}$ & & $\begin{array}{l}0.000 \\
(0.544)\end{array}$ & \\
\hline University & $-0.542^{* * *}$ & & $-0.626^{* * *}$ & & $-0.533^{* * *}$ & \\
\hline $\begin{array}{l}\text { degree } \\
\text { Vocational }\end{array}$ & $\begin{array}{l}(8.641) \\
-0.327 * * *\end{array}$ & & $\begin{array}{l}(3.912) \\
-0.403 * * *\end{array}$ & & $\begin{array}{l}(3.528) \\
-0.338 * * *\end{array}$ & \\
\hline qualifications & (6.332) & & (3.219) & & (2.888) & \\
\hline A - levels plus & $\begin{array}{l}-0.288^{* * *} \\
(5.034)\end{array}$ & & $\begin{array}{l}-0.438^{* * *} \\
(3.134)\end{array}$ & & $\begin{array}{l}-0.377^{* * *} \\
(2.864)\end{array}$ & \\
\hline O - levels plus & $\begin{array}{l}-0.200^{* * *} \\
(3.791)\end{array}$ & & $\begin{array}{l}-0.292^{* *} \\
(2.289)\end{array}$ & & $\begin{array}{l}-0.245^{* *} \\
(2.060)\end{array}$ & \\
\hline $\begin{array}{l}\text { Commercial or } \\
\text { Apprentice }\end{array}$ & $\begin{array}{l}-0.165^{* *} \\
(2.519)\end{array}$ & & $\begin{array}{l}-0.163 \\
(1.058)\end{array}$ & & $\begin{array}{l}-0.246^{*} \\
(1.721)\end{array}$ & \\
\hline Married & $\begin{array}{l}0.116^{* * *} \\
(3.791)\end{array}$ & $\begin{array}{l}0.188^{* * *} \\
(6.612)\end{array}$ & $\begin{array}{l}0.118 \\
(1.467)\end{array}$ & $\begin{array}{l}0.243 * * * \\
(3.237)\end{array}$ & $\begin{array}{l}0.130 * \\
(1.692)\end{array}$ & $\begin{array}{l}0.253 * * * \\
(3.540)\end{array}$ \\
\hline Mortgage house & $\begin{array}{l}-0.100 * * * \\
(2.986)\end{array}$ & $\begin{array}{l}-0.117^{* * *} \\
(3.487)\end{array}$ & $\begin{array}{l}-0.072 \\
(0.698)\end{array}$ & $\begin{array}{l}-0.134 \\
(1.303)\end{array}$ & $\begin{array}{l}-0.076 \\
(0.773)\end{array}$ & $\begin{array}{l}-0.131 \\
(1.343)\end{array}$ \\
\hline $\begin{array}{l}\text { Paid outright } \\
\text { house }\end{array}$ & $\begin{array}{l}-0.060 \\
(1.236)\end{array}$ & $\begin{array}{l}-0.011 \\
(0.240)\end{array}$ & $\begin{array}{l}-0.039 \\
(0.301)\end{array}$ & $\begin{array}{l}-0.019 \\
(0.145)\end{array}$ & $\begin{array}{l}-0.030 \\
(0.241)\end{array}$ & $\begin{array}{l}-0.006 \\
(0.049)\end{array}$ \\
\hline $\begin{array}{l}\text { Promotion } \\
\text { Opportunities }\end{array}$ & $\begin{array}{l}0.292 * * * \\
(11.336)\end{array}$ & $\begin{array}{l}0.264 * * * \\
(10.366)\end{array}$ & $\begin{array}{l}0.261 * * * \\
(3.700)\end{array}$ & $\begin{array}{l}0.217^{* * *} \\
(3.090)\end{array}$ & $\begin{array}{l}0.272^{* * *} \\
(4.102)\end{array}$ & $\begin{array}{l}0.232^{* * *} \\
(3.523)\end{array}$ \\
\hline Public Sector & $\begin{array}{l}-0.056 \\
(1.268)\end{array}$ & $\begin{array}{l}-0.057 \\
(1.304)\end{array}$ & $\begin{array}{l}0.105 \\
(0.884)\end{array}$ & $\begin{array}{l}0.092 \\
(0.776)\end{array}$ & $\begin{array}{l}0.045 \\
(0.403)\end{array}$ & $\begin{array}{l}0.034 \\
(0.297)\end{array}$ \\
\hline $\begin{array}{l}\text { Works } \\
\text { mornings only }\end{array}$ & $\begin{array}{l}0.119 * \\
(1.915)\end{array}$ & $\begin{array}{l}0.149 * * \\
(2.381)\end{array}$ & $\begin{array}{l}0.172 \\
(1.140)\end{array}$ & $\begin{array}{l}0.254^{*} \\
(1.691)\end{array}$ & $\begin{array}{l}0.159 \\
(1.093)\end{array}$ & $\begin{array}{l}0.226 \\
(1.551)\end{array}$ \\
\hline $\begin{array}{l}\text { Works evenings } \\
\text { or nights }\end{array}$ & $\begin{array}{l}-0.139 * * \\
(2.328)\end{array}$ & $\begin{array}{l}-0.139 * * \\
(2.322)\end{array}$ & $\begin{array}{l}-0.019 \\
(0.126)\end{array}$ & $\begin{array}{l}-0.051 \\
(0.335)\end{array}$ & $\begin{array}{l}-0.065 \\
(0.444)\end{array}$ & $\begin{array}{l}-0.091 \\
(0.620)\end{array}$ \\
\hline $\begin{array}{l}\text { Variable shift } \\
\text { patterns }\end{array}$ & $\begin{array}{l}-0.033 \\
(0.875)\end{array}$ & $\begin{array}{l}-0.024 \\
(0.646)\end{array}$ & $\begin{array}{l}0.088 \\
(0.927)\end{array}$ & $\begin{array}{l}0.082 \\
(0.863)\end{array}$ & $\begin{array}{l}0.033 \\
(0.373)\end{array}$ & $\begin{array}{l}0.030 \\
(0.333)\end{array}$ \\
\hline Fair health & $\begin{array}{l}0.117^{* *} \\
(2.516)\end{array}$ & $\begin{array}{l}0.113^{* *} \\
(2.429)\end{array}$ & $\begin{array}{l}0.092 \\
(0.735)\end{array}$ & $\begin{array}{l}0.092 \\
(0.731)\end{array}$ & $\begin{array}{l}0.077 \\
(0.638)\end{array}$ & $\begin{array}{l}0.077 \\
(0.638)\end{array}$ \\
\hline Good health & $\begin{array}{l}0.297 * * * \\
(6.474)\end{array}$ & $\begin{array}{l}0.282 * * * \\
(6.139)\end{array}$ & $\begin{array}{l}0.292 * * \\
(2.417)\end{array}$ & $\begin{array}{l}0.267^{* *} \\
(2.206)\end{array}$ & $\begin{array}{l}0.294^{* *} \\
(2.542)\end{array}$ & $\begin{array}{l}0.273 * * \\
(2.350)\end{array}$ \\
\hline Excellent health & $\begin{array}{l}0.520 * * * \\
(10.471)\end{array}$ & $\begin{array}{l}0.494 * * * \\
(9.950)\end{array}$ & $\begin{array}{l}0.485^{* * * *} \\
(3.805)\end{array}$ & $\begin{array}{l}0.441 * * * \\
(3.450)\end{array}$ & $\begin{array}{l}0.492^{* * * *} \\
(4.028)\end{array}$ & $\begin{array}{l}0.457 * * * \\
(3.736)\end{array}$ \\
\hline $\begin{array}{l}\text { Trade union } \\
\text { member }\end{array}$ & $\begin{array}{l}-0.217^{* * * *} \\
(6.768)\end{array}$ & $\begin{array}{l}-0.212^{* * * *} \\
(6.595)\end{array}$ & $\begin{array}{l}-0.344^{* * * *} \\
(4.282)\end{array}$ & $\begin{array}{l}-0.343^{* * *} \\
(4.271)\end{array}$ & $\begin{array}{l}-0.288 * * * \\
(3.793)\end{array}$ & $\begin{array}{l}-0.284 * * * \\
(3.735)\end{array}$ \\
\hline Temporary Job & $\begin{array}{l}-0.267 * * * \\
(5.068)\end{array}$ & $\begin{array}{l}-0.282^{* * * *} \\
(5.370)\end{array}$ & $\begin{array}{l}-0.427^{* * * *} \\
(3.031)\end{array}$ & $\begin{array}{l}-0.449 * * * \\
(3.183)\end{array}$ & $\begin{array}{l}-0.420 * * * \\
(3.081)\end{array}$ & $\begin{array}{l}-0.447 * * * \\
(3.270)\end{array}$ \\
\hline Incremental pay & $\begin{array}{l}0.203^{* * *} \\
(7.968)\end{array}$ & $\begin{array}{l}0.194 * * * \\
(7.624)\end{array}$ & $\begin{array}{l}0.307 * * * \\
(4.440)\end{array}$ & $\begin{array}{l}0.287 * * * \\
(4.139)\end{array}$ & $\begin{array}{l}0.292^{* * *} \\
(4.456)\end{array}$ & $\begin{array}{l}0.271^{* * *} \\
(4.132)\end{array}$ \\
\hline Part Time & $\begin{array}{l}-0.139 * * * \\
(2.617)\end{array}$ & $\begin{array}{l}-0.125^{* *} \\
(2.328)\end{array}$ & $\begin{array}{l}-0.115 \\
(0.829)\end{array}$ & $\begin{array}{l}-0.146 \\
(1.050)\end{array}$ & $\begin{array}{l}-0.218^{*} \\
(1.654)\end{array}$ & $\begin{array}{l}-0.244^{*} \\
(1.833)\end{array}$ \\
\hline
\end{tabular}




\begin{tabular}{|c|c|c|c|c|c|c|}
\hline Travel Time & $\begin{array}{l}-0.002 * * \\
(2.517)\end{array}$ & $\begin{array}{l}-0.002^{* * *} \\
(2.891)\end{array}$ & $\begin{array}{l}-0.004 * * \\
(2.032)\end{array}$ & $\begin{array}{l}-0.004 * * \\
(2.130)\end{array}$ & $\begin{array}{l}-0.004 * * \\
(2.153)\end{array}$ & $\begin{array}{l}-0.004^{* *} \\
(2.246)\end{array}$ \\
\hline Size 25 - 99 & $\begin{array}{l}-0.156^{* * *} \\
(4.888)\end{array}$ & $\begin{array}{l}-0.148^{* * * *} \\
(4.638)\end{array}$ & $\begin{array}{l}-0.187 * * \\
(2.150)\end{array}$ & $\begin{array}{l}-0.167^{*} \\
(1.903)\end{array}$ & $\begin{array}{l}-0.196 * * \\
(2.384)\end{array}$ & $\begin{array}{l}-0.181^{* *} \\
(2.184)\end{array}$ \\
\hline Size 100 - 499 & $\begin{array}{l}-0.217^{* * *} \\
(6.193)\end{array}$ & $\begin{array}{l}-0.207^{* * * *} \\
(5.840)\end{array}$ & $\begin{array}{l}-0.088 \\
(0.905)\end{array}$ & $\begin{array}{l}-0.083 \\
(0.833)\end{array}$ & $\begin{array}{l}-0.108 \\
(1.163)\end{array}$ & $\begin{array}{l}-0.100 \\
(1.064)\end{array}$ \\
\hline Size 500 plus & $\begin{array}{l}-0.199 * * * \\
(5.136)\end{array}$ & $\begin{array}{l}-0.190^{* * *} \\
(4.871)\end{array}$ & $\begin{array}{l}-0.206 * \\
(1.937)\end{array}$ & $\begin{array}{l}-0.207^{*} \\
(1.916)\end{array}$ & $\begin{array}{l}-0.207 * * \\
(2.048)\end{array}$ & $\begin{array}{l}-0.209 * * \\
(2.034)\end{array}$ \\
\hline $\begin{array}{l}\text { Voluntarily } \\
\text { Quit }\end{array}$ & $\begin{array}{l}0.321 * * * \\
(8.008)\end{array}$ & $\begin{array}{l}0.315^{* * *} \\
(7.857)\end{array}$ & $\begin{array}{l}0.237^{*} \\
(1.917)\end{array}$ & $\begin{array}{l}0.228^{*} \\
(1.833)\end{array}$ & $\begin{array}{l}0.228 * * \\
(1.977)\end{array}$ & $\begin{array}{l}0.217^{*} \\
(1.877)\end{array}$ \\
\hline $\begin{array}{l}\text { Number of } \\
\text { weeks not } \\
\text { employed in the } \\
\text { last year }\end{array}$ & $\begin{array}{l}0.005^{* * *} \\
(4.077)\end{array}$ & $\begin{array}{l}0.004^{* * * *} \\
(3.398)\end{array}$ & $\begin{array}{l}0.009 * * * \\
(2.839)\end{array}$ & $\begin{array}{l}0.008 * * \\
(2.514)\end{array}$ & $\begin{array}{l}0.008^{* * *} \\
(2.615)\end{array}$ & $\begin{array}{l}0.007 * * \\
(2.312)\end{array}$ \\
\hline Wave10 & $\begin{array}{l}-0.088^{* * * *} \\
(3.757)\end{array}$ & $\begin{array}{l}-0.085^{* * *} \\
(3.632)\end{array}$ & $\begin{array}{l}0.046 \\
(0.725)\end{array}$ & $\begin{array}{l}0.051 \\
(0.798)\end{array}$ & $\begin{array}{l}0.056 \\
(0.929)\end{array}$ & $\begin{array}{l}0.062 \\
(1.030)\end{array}$ \\
\hline Wave11 & $\begin{array}{l}-0.046^{*} \\
(1.776)\end{array}$ & $\begin{array}{l}-0.027 \\
(1.027)\end{array}$ & $\begin{array}{l}0.092 \\
(1.240)\end{array}$ & $\begin{array}{l}0.096 \\
(1.260)\end{array}$ & $\begin{array}{l}0.092 \\
(1.306)\end{array}$ & $\begin{array}{l}0.097 \\
(1.353)\end{array}$ \\
\hline Rest of England & $\begin{array}{l}-0.169 * * * \\
(4.314)\end{array}$ & $\begin{array}{l}-0.175^{* * *} \\
(4.458)\end{array}$ & & & & \\
\hline $\begin{array}{l}\text { London and } \\
\text { South East }\end{array}$ & $\begin{array}{l}-0.291 * * * \\
(6.337)\end{array}$ & $\begin{array}{l}-0.254^{* * *} \\
(5.424)\end{array}$ & & & & \\
\hline Scotland & $\begin{array}{l}-0.213^{* * *} \\
(4.929)\end{array}$ & $\begin{array}{l}-0.221^{* * *} \\
(5.108)\end{array}$ & & & & \\
\hline $\begin{array}{l}\text { Log real } \\
\text { comparative } \\
\text { wage }\end{array}$ & & $\begin{array}{l}-0.364 * * * \\
(3.399)\end{array}$ & & $\begin{array}{l}-0.105 \\
(0.364)\end{array}$ & & $\begin{array}{l}-0.035 \\
(0.128)\end{array}$ \\
\hline $\begin{array}{l}\text { Objective } 1 \\
\text { area }\end{array}$ & & & $\begin{array}{l}0.122^{*} \\
(1.690)\end{array}$ & $\begin{array}{l}0.119 * \\
(1.646)\end{array}$ & & \\
\hline Cut 1 & $\begin{array}{l}-4.71 * * * \\
(17.41)\end{array}$ & $\begin{array}{l}-4.35 * * * \\
(19.73)\end{array}$ & $\begin{array}{l}-4.20 * * * \\
(5.93)\end{array}$ & $\begin{array}{l}-4.29 * * * \\
(7.24)\end{array}$ & $\begin{array}{l}-3.99 * * * \\
(5.93)\end{array}$ & $\begin{array}{l}-4.11^{* * *} \\
(7.32)\end{array}$ \\
\hline Cut 2 & $\begin{array}{l}-4.07 * * * \\
(-15.14)\end{array}$ & $\begin{array}{l}-3.71 * * * \\
(-16.97)\end{array}$ & $\begin{array}{l}-3.54 * * * \\
(-5.05)\end{array}$ & $\begin{array}{l}-3.63 * * * \\
(-6.22)\end{array}$ & $\begin{array}{l}-3.35 * * * \\
(-5.03)\end{array}$ & $\begin{array}{l}-3.47 * * * \\
(-6.27)\end{array}$ \\
\hline Cut 3 & $\begin{array}{l}-3.37 * * * \\
(-12.59)\end{array}$ & $\begin{array}{l}-3.01^{* * *} \\
(-13.85)\end{array}$ & $\begin{array}{l}-2.91 * * * \\
(-4.18)\end{array}$ & $\begin{array}{l}-3.00 * * * \\
(-5.19)\end{array}$ & $\begin{array}{l}-2.71 * * * \\
(-4.10)\end{array}$ & $\begin{array}{l}-2.84 * * * \\
(-5.17)\end{array}$ \\
\hline Cut 4 & $\begin{array}{l}-2.93 * * * \\
(-10.96)\end{array}$ & $\begin{array}{l}-2.57 * * * \\
(-11.85)\end{array}$ & $\begin{array}{l}-2.47 * * * \\
(-3.57)\end{array}$ & $\begin{array}{l}-2.57 * * * \\
(-4.47)\end{array}$ & $\begin{array}{l}-2.27 * * * \\
(-3.44)\end{array}$ & $\begin{array}{l}-2.40^{* * *} \\
(-4.39)\end{array}$ \\
\hline Cut 5 & $\begin{array}{l}-1.97 * * * \\
(-7.42)\end{array}$ & $\begin{array}{l}-1.61 * * * \\
(-7.50)\end{array}$ & $\begin{array}{l}-1.52^{* *} \\
(-2.20)\end{array}$ & $\begin{array}{l}-1.62 * * * \\
(-2.84)\end{array}$ & $\begin{array}{l}-1.33^{* *} \\
(-2.03)\end{array}$ & $\begin{array}{l}-1.46^{* * *} \\
(-2.70)\end{array}$ \\
\hline Cut 6 & $\begin{array}{l}0.08 \\
(0.33)\end{array}$ & $\begin{array}{l}0.44^{* *} \\
(2.05)\end{array}$ & $\begin{array}{l}0.53 \\
(0.78)\end{array}$ & $\begin{array}{l}0.42 \\
(0.75)\end{array}$ & $\begin{array}{l}0.68 \\
(1.05)\end{array}$ & $\begin{array}{l}0.55 \\
(1.03)\end{array}$ \\
\hline Log likelihood & -22334 & -22388 & -3052 & -3069 & -3333 & -3349 \\
\hline Observations & 15916 & 15911 & 2230 & 2229 & 2429 & 2428 \\
\hline
\end{tabular}




\begin{tabular}{|c|c|c|c|c|c|c|}
\hline & Scot & and & $\begin{array}{r}\text { London } \\
\mathbf{E}\end{array}$ & $\begin{array}{l}\text { nd South } \\
\text { st }\end{array}$ & Rest of & igland \\
\hline & (7) & (8) & (9) & (10) & (11) & (12) \\
\hline Gender & $\begin{array}{l}-0.204^{* * *} \\
(3.172)\end{array}$ & $\begin{array}{l}-0.170 * * * \\
(2.638)\end{array}$ & $\begin{array}{l}-0.259 * * * \\
(3.801)\end{array}$ & $\begin{array}{l}-0.246^{* * *} \\
(3.597)\end{array}$ & $\begin{array}{l}-0.241^{* * *} \\
(4.542)\end{array}$ & $\begin{array}{l}-0.231 * * * \\
(4.349)\end{array}$ \\
\hline Log usual real gross & $0.138 *$ & 0.088 & $0.155^{* *}$ & 0.105 & $0.239 * * *$ & $0.169 * * *$ \\
\hline hourly wage & $(1.922)$ & (1.282) & (2.192) & $(1.569)$ & (4.234) & (3.104) \\
\hline Log usual hours & $\begin{array}{l}-0.049 \\
(0.431)\end{array}$ & $\begin{array}{l}-0.066 \\
(0.576)\end{array}$ & $\begin{array}{l}-0.219 * * \\
(2.026)\end{array}$ & $\begin{array}{l}-0.215^{* *} \\
(1.983)\end{array}$ & $\begin{array}{l}-0.367 * * * \\
(4.512)\end{array}$ & $\begin{array}{l}-0.389 * * * \\
(4.783)\end{array}$ \\
\hline Job Tenure & $\begin{array}{l}-0.002^{*} \\
(1.946)\end{array}$ & $\begin{array}{l}-0.001 \\
(1.431)\end{array}$ & $\begin{array}{l}-0.003 * * \\
(2.483)\end{array}$ & $\begin{array}{l}-0.002 * \\
(1.774)\end{array}$ & $\begin{array}{l}-0.002 * * \\
(2.206)\end{array}$ & $\begin{array}{l}-0.001 \\
(1.292)\end{array}$ \\
\hline Tenure Squared & $\begin{array}{l}0.000 \\
(1.073)\end{array}$ & $\begin{array}{l}0.000 \\
(1.572)\end{array}$ & $\begin{array}{l}0.000 * * \\
(2.435)\end{array}$ & $\begin{array}{l}0.000 * * \\
(2.044)\end{array}$ & $\begin{array}{l}0.000 \\
(0.705)\end{array}$ & $\begin{array}{l}0.000 \\
(0.570)\end{array}$ \\
\hline Age & $\begin{array}{l}-0.068 * * * \\
(3.646)\end{array}$ & & $\begin{array}{l}0.001 \\
(0.038)\end{array}$ & & $\begin{array}{l}-0.017 \\
(1.201)\end{array}$ & \\
\hline Age square & $\begin{array}{l}0.001^{* * *} \\
(4.339)\end{array}$ & & $\begin{array}{l}0.000 \\
(0.066)\end{array}$ & & $\begin{array}{l}0.000 \\
(1.461)\end{array}$ & \\
\hline University degree & $\begin{array}{l}-0.269 * * \\
(2.062)\end{array}$ & & $\begin{array}{l}-0.629 * * * \\
(4.472)\end{array}$ & & $\begin{array}{l}-0.678 * * * \\
(6.536)\end{array}$ & \\
\hline $\begin{array}{l}\text { Vocational } \\
\text { qualifications }\end{array}$ & $\begin{array}{l}-0.159 \\
(1.448)\end{array}$ & & $\begin{array}{l}-0.456^{* * *} \\
(3.688)\end{array}$ & & $\begin{array}{l}-0.349 * * * \\
(4.204)\end{array}$ & \\
\hline A - levels plus & $\begin{array}{l}-0.087 \\
(0.752)\end{array}$ & & $\begin{array}{l}-0.315^{* *} \\
(2.291)\end{array}$ & & $\begin{array}{l}-0.337 * * * \\
(3.585)\end{array}$ & \\
\hline O - levels plus & $\begin{array}{l}-0.003 \\
(0.027)\end{array}$ & & $\begin{array}{l}-0.456 * * * \\
(3.530)\end{array}$ & & $\begin{array}{l}-0.178 * * \\
(2.101)\end{array}$ & \\
\hline Commercial or & -0.049 & & -0.241 & & $-0.198 *$ & \\
\hline Apprentice & $(0.311)$ & & $(1.548)$ & & $(1.940)$ & \\
\hline Married & $\begin{array}{l}0.102 \\
(1.588)\end{array}$ & $\begin{array}{l}0.174 * * * \\
(2.901)\end{array}$ & $\begin{array}{l}0.068 \\
(1.016)\end{array}$ & $\begin{array}{l}0.118^{*} \\
(1.933)\end{array}$ & $\begin{array}{l}0.143^{* * * *} \\
(2.926)\end{array}$ & $\begin{array}{l}0.207 * * * \\
(4.521)\end{array}$ \\
\hline Mortgage house & $\begin{array}{l}-0.116^{*} \\
(1.692)\end{array}$ & $\begin{array}{l}-0.121^{*} \\
(1.760)\end{array}$ & $\begin{array}{l}0.082 \\
(1.193)\end{array}$ & $\begin{array}{l}0.070 \\
(1.032)\end{array}$ & $\begin{array}{l}-0.204 * * * \\
(3.753)\end{array}$ & $\begin{array}{l}-0.222 * * * \\
(4.098)\end{array}$ \\
\hline Paid outright house & $\begin{array}{l}0.053 \\
(0.474)\end{array}$ & $\begin{array}{l}0.161 \\
(1.456)\end{array}$ & $\begin{array}{l}0.162 * \\
(1.660)\end{array}$ & $\begin{array}{l}0.185^{*} \\
(1.934)\end{array}$ & $\begin{array}{l}-0.229 * * * \\
(2.943)\end{array}$ & $\begin{array}{l}-0.201 * * * \\
(2.647)\end{array}$ \\
\hline $\begin{array}{l}\text { Promotion } \\
\text { Opportunities }\end{array}$ & $\begin{array}{l}0.243 * * * \\
(4.327)\end{array}$ & $\begin{array}{l}0.191 * * * \\
(3.451)\end{array}$ & $\begin{array}{l}0.216 * * * \\
(3.941)\end{array}$ & $\begin{array}{l}0.205 * * * \\
(3.774)\end{array}$ & $\begin{array}{l}0.369 * * * \\
(9.137)\end{array}$ & $\begin{array}{l}0.352 * * * \\
(8.767)\end{array}$ \\
\hline Public Sector & $\begin{array}{l}-0.007 \\
(0.074)\end{array}$ & $\begin{array}{l}0.018 \\
(0.189)\end{array}$ & $\begin{array}{l}-0.125 \\
(1.372)\end{array}$ & $\begin{array}{l}-0.143 \\
(1.576)\end{array}$ & $\begin{array}{l}-0.062 \\
(0.870)\end{array}$ & $\begin{array}{l}-0.070 \\
(0.972)\end{array}$ \\
\hline Works mornings only & $\begin{array}{l}0.232 \\
(1.574)\end{array}$ & $\begin{array}{l}0.226 \\
(1.526)\end{array}$ & $\begin{array}{l}0.005 \\
(0.041)\end{array}$ & $\begin{array}{l}0.053 \\
(0.401)\end{array}$ & $\begin{array}{l}0.115 \\
(1.170)\end{array}$ & $\begin{array}{l}0.132 \\
(1.339)\end{array}$ \\
\hline $\begin{array}{l}\text { Works evenings or } \\
\text { nights }\end{array}$ & $\begin{array}{l}-0.032 \\
(0.259)\end{array}$ & $\begin{array}{l}-0.041 \\
(0.332)\end{array}$ & $\begin{array}{l}-0.258 * \\
(1.735)\end{array}$ & $\begin{array}{l}-0.248 * \\
(1.671)\end{array}$ & $\begin{array}{l}-0.185^{* *} \\
(2.025)\end{array}$ & $\begin{array}{l}-0.183 * * \\
(1.999)\end{array}$ \\
\hline Variable shift patterns & $\begin{array}{l}-0.015 \\
(0.196)\end{array}$ & $\begin{array}{l}-0.016 \\
(0.207)\end{array}$ & $\begin{array}{l}-0.029 \\
(0.314)\end{array}$ & $\begin{array}{l}-0.022 \\
(0.237)\end{array}$ & $\begin{array}{l}-0.060 \\
(1.004)\end{array}$ & $\begin{array}{l}-0.047 \\
(0.786)\end{array}$ \\
\hline Fair health & $\begin{array}{l}0.040 \\
(0.371)\end{array}$ & $\begin{array}{l}0.032 \\
(0.294)\end{array}$ & $\begin{array}{l}0.193^{*} \\
(1.886)\end{array}$ & $\begin{array}{l}0.188^{*} \\
(1.833)\end{array}$ & $\begin{array}{l}0.134^{*} \\
(1.930)\end{array}$ & $\begin{array}{l}0.132 * \\
(1.898)\end{array}$ \\
\hline Good health & $\begin{array}{l}0.195^{*} \\
(1.854)\end{array}$ & $\begin{array}{l}0.187^{*} \\
(1.768)\end{array}$ & $\begin{array}{l}0.439 * * * \\
(4.333)\end{array}$ & $\begin{array}{l}0.415 * * * \\
(4.102)\end{array}$ & $\begin{array}{l}0.282 * * * \\
(4.050)\end{array}$ & $\begin{array}{l}0.272 * * * \\
(3.907)\end{array}$ \\
\hline Excellent health & $\begin{array}{l}0.483 * * * \\
(4.316)\end{array}$ & $\begin{array}{l}0.451 * * * \\
(4.026)\end{array}$ & $\begin{array}{l}0.662 * * * \\
(6.010)\end{array}$ & $\begin{array}{l}0.636^{* * * *} \\
(5.782)\end{array}$ & $\begin{array}{l}0.481 * * * \\
(6.265)\end{array}$ & $\begin{array}{l}0.460 * * * \\
(5.996)\end{array}$ \\
\hline Trade union member & $\begin{array}{l}-0.245^{* * *} \\
(3.645)\end{array}$ & $\begin{array}{l}-0.235^{* * *} \\
(3.479)\end{array}$ & $\begin{array}{l}-0.217^{* * *} \\
(2.954)\end{array}$ & $\begin{array}{l}-0.216^{* * *} \\
(2.930)\end{array}$ & $\begin{array}{l}-0.177 * * * \\
(3.417)\end{array}$ & $\begin{array}{l}-0.167 * * * \\
(3.240)\end{array}$ \\
\hline Temporary Job & $\begin{array}{l}-0.325^{* * *} \\
(2.844)\end{array}$ & $\begin{array}{l}-0.312^{* * *} \\
(2.733)\end{array}$ & $\begin{array}{l}-0.191^{*} \\
(1.789)\end{array}$ & $\begin{array}{l}-0.210^{* *} \\
(1.980)\end{array}$ & $\begin{array}{l}-0.225^{* * *} \\
(2.615)\end{array}$ & $\begin{array}{l}-0.248 * * * \\
(2.888)\end{array}$ \\
\hline Incremental pay & $\begin{array}{l}0.255^{* * * *} \\
(4.624)\end{array}$ & $\begin{array}{l}0.246 * * * \\
(4.452)\end{array}$ & $\begin{array}{l}0.244 * * * \\
(4.473)\end{array}$ & $\begin{array}{l}0.240 * * * \\
(4.400)\end{array}$ & $\begin{array}{l}0.125 * * * \\
(3.142)\end{array}$ & $\begin{array}{l}0.119 * * * \\
(2.981)\end{array}$ \\
\hline Part Time & $\begin{array}{l}-0.380^{* * *} \\
(3.359)\end{array}$ & $\begin{array}{l}-0.336^{* * *} \\
(2.915)\end{array}$ & $\begin{array}{l}-0.120 \\
(1.010)\end{array}$ & $\begin{array}{l}-0.122 \\
(1.016)\end{array}$ & $\begin{array}{l}0.037 \\
(0.445)\end{array}$ & $\begin{array}{l}0.058 \\
(0.683)\end{array}$ \\
\hline Travel Time & $\begin{array}{l}-0.004^{* * *} \\
(2.619)\end{array}$ & $\begin{array}{l}-0.004^{* * *} \\
(2.649)\end{array}$ & $\begin{array}{l}-0.000 \\
(0.053)\end{array}$ & $\begin{array}{l}-0.000 \\
(0.255)\end{array}$ & $\begin{array}{l}-0.001 \\
(1.237)\end{array}$ & $\begin{array}{l}-0.002 \\
(1.475)\end{array}$ \\
\hline
\end{tabular}




\begin{tabular}{|c|c|c|c|c|c|c|}
\hline Size 25 - 99 & $\begin{array}{l}-0.061 \\
(0.901)\end{array}$ & $\begin{array}{l}-0.041 \\
(0.599)\end{array}$ & $\begin{array}{l}-0.093 \\
(1.365)\end{array}$ & $\begin{array}{l}-0.089 \\
(1.291)\end{array}$ & $\begin{array}{l}-0.231^{* * *} \\
(4.596)\end{array}$ & $\begin{array}{l}-0.229 * * * \\
(4.527)\end{array}$ \\
\hline Size 100 - 499 & $\begin{array}{l}-0.200^{* * *} \\
(2.652)\end{array}$ & $\begin{array}{l}-0.190 * * \\
(2.490)\end{array}$ & $\begin{array}{l}-0.201^{* * *} \\
(2.662)\end{array}$ & $\begin{array}{l}-0.186 * * \\
(2.437)\end{array}$ & $\begin{array}{l}-0.281^{* * *} \\
(5.148)\end{array}$ & $\begin{array}{l}-0.273 * * * \\
(4.952)\end{array}$ \\
\hline Size 500 plus & $\begin{array}{l}-0.131 \\
(1.636)\end{array}$ & $\begin{array}{l}-0.108 \\
(1.340)\end{array}$ & $\begin{array}{l}-0.152 * \\
(1.859)\end{array}$ & $\begin{array}{l}-0.137 * \\
(1.657)\end{array}$ & $\begin{array}{l}-0.263 * * * \\
(4.157)\end{array}$ & $\begin{array}{l}-0.250 * * * \\
(3.931)\end{array}$ \\
\hline Voluntarily Quit & $\begin{array}{l}0.241 * * * \\
(2.741)\end{array}$ & $\begin{array}{l}0.228 * * * \\
(2.586)\end{array}$ & $\begin{array}{l}0.355 * * * \\
(4.406)\end{array}$ & $\begin{array}{l}0.353 * * * \\
(4.389)\end{array}$ & $\begin{array}{l}0.382 * * * \\
(6.122)\end{array}$ & $\begin{array}{l}0.380 * * * \\
(6.082)\end{array}$ \\
\hline $\begin{array}{l}\text { Number of weeks not } \\
\text { employed in the last } \\
\text { year }\end{array}$ & $\begin{array}{l}0.004 \\
(1.573)\end{array}$ & $\begin{array}{l}0.003 \\
(1.321)\end{array}$ & $\begin{array}{l}0.005 * * \\
(2.110)\end{array}$ & $\begin{array}{l}0.004^{*} \\
(1.874)\end{array}$ & $\begin{array}{l}0.004 * * \\
(2.021)\end{array}$ & $\begin{array}{l}0.003 \\
(1.534)\end{array}$ \\
\hline Wave10 & $\begin{array}{l}-0.103 * * \\
(2.007)\end{array}$ & $\begin{array}{l}-0.093^{*} \\
(1.802)\end{array}$ & $\begin{array}{l}-0.127 * * \\
(2.526)\end{array}$ & $\begin{array}{l}-0.130 * * * \\
(2.579)\end{array}$ & $\begin{array}{l}-0.109 * * * \\
(3.016)\end{array}$ & $\begin{array}{l}-0.108^{* * *} \\
(2.993)\end{array}$ \\
\hline Wave11 & $\begin{array}{l}-0.086 \\
(1.430)\end{array}$ & $\begin{array}{l}-0.059 \\
(0.978)\end{array}$ & $\begin{array}{l}-0.065 \\
(1.241)\end{array}$ & $\begin{array}{l}-0.058 \\
(1.094)\end{array}$ & $\begin{array}{l}-0.062 \\
(1.587)\end{array}$ & $\begin{array}{l}-0.039 \\
(0.979)\end{array}$ \\
\hline $\begin{array}{l}\text { Log real } \\
\text { comparative wage }\end{array}$ & & $\begin{array}{l}-0.473 * \\
(1.915)\end{array}$ & & $\begin{array}{l}-0.275 \\
(1.167)\end{array}$ & & $\begin{array}{l}-0.452 * * * \\
(2.786)\end{array}$ \\
\hline Cut 1 & $\begin{array}{l}-4.86 * * * \\
(8.35)\end{array}$ & $\begin{array}{l}-3.94^{* * * *} \\
(8.17)\end{array}$ & $\begin{array}{l}-4.16 * * * \\
(6.97)\end{array}$ & $\begin{array}{l}-3.93 * * * \\
(8.10)\end{array}$ & $\begin{array}{l}-4.84 * * * \\
(11.37)\end{array}$ & $\begin{array}{l}-4.57 * * * \\
(13.36)\end{array}$ \\
\hline Cut 2 & $\begin{array}{l}-4.14^{* * *} \\
(-7.20)\end{array}$ & $\begin{array}{l}-3.23^{* * *} \\
(-6.77)\end{array}$ & $\begin{array}{l}-3.49 * * * \\
(-5.88)\end{array}$ & $\begin{array}{l}-3.26 * * * \\
(-6.77)\end{array}$ & $\begin{array}{l}-4.24 * * * \\
(-10.01)\end{array}$ & $\begin{array}{l}-3.97 * * * \\
(-11.69)\end{array}$ \\
\hline Cut 3 & $\begin{array}{l}-3.45 * * * \\
(-6.03)\end{array}$ & $\begin{array}{l}-2.53^{* * *} \\
(-5.36)\end{array}$ & $\begin{array}{l}-2.74 * * * \\
(-4.64)\end{array}$ & $\begin{array}{l}-2.51 * * * \\
(-5.25)\end{array}$ & $\begin{array}{l}-3.53 * * * \\
(-8.37)\end{array}$ & $\begin{array}{l}-3.26 * * * \\
(-9.65)\end{array}$ \\
\hline Cut 4 & $\begin{array}{l}-3.01 * * * \\
(-5.28)\end{array}$ & $\begin{array}{l}-2.10 * * * \\
(-4.45)\end{array}$ & $\begin{array}{l}-2.32 * * * \\
(-3.93)\end{array}$ & $\begin{array}{l}-2.09 * * * \\
(-4.38)\end{array}$ & $\begin{array}{l}-3.07 * * * \\
(-7.29)\end{array}$ & $\begin{array}{l}-2.80 * * * \\
(-8.31)\end{array}$ \\
\hline Cut 5 & $\begin{array}{l}-2.09 * * * \\
(-3.69)\end{array}$ & $\begin{array}{l}-1.18 * * \\
(-2.51)\end{array}$ & $\begin{array}{l}-1.40 * * \\
(-2.38)\end{array}$ & $\begin{array}{l}-1.17 * * \\
(-2.47)\end{array}$ & $\begin{array}{l}-2.06 * * * \\
(-4.92)\end{array}$ & $\begin{array}{l}-1.80 * * * \\
(-5.37)\end{array}$ \\
\hline Cut 6 & $\begin{array}{l}0.01 \\
(0.02)\end{array}$ & $\begin{array}{l}0.91^{*} \\
(1.94)\end{array}$ & $\begin{array}{l}0.70 \\
(1.19)\end{array}$ & $\begin{array}{l}0.91^{*} \\
(1.93)\end{array}$ & $\begin{array}{l}0.01 \\
(0.02)\end{array}$ & $\begin{array}{l}0.26 \\
(0.79)\end{array}$ \\
\hline Log likelihood & -4819 & -4837 & -4725 & -4737 & -9350 & -9374 \\
\hline Observations & 3403 & 3402 & 3392 & 3391 & 6692 & 6690 \\
\hline
\end{tabular}


Table 3B: OVERALL JOB SATISFACTION - MALES

\begin{tabular}{|c|c|c|c|c|c|c|}
\hline & \multicolumn{2}{|c|}{ National } & \multicolumn{2}{|c|}{$\begin{array}{l}\text { WALES with } \\
\text { objective } 1 \text { variable }\end{array}$} & \multicolumn{2}{|c|}{$\begin{array}{l}\text { WALES without } \\
\text { objective } 1 \text { variable }\end{array}$} \\
\hline & (1) & (2) & (3) & (4) & (5) & (6) \\
\hline $\begin{array}{l}\text { Log usual real gross } \\
\text { hourly wage }\end{array}$ & $\begin{array}{l}0.328 * * * \\
(6.282)\end{array}$ & $\begin{array}{l}0.264^{* * *} \\
(5.306)\end{array}$ & $\begin{array}{l}0.331^{* *} \\
(2.203)\end{array}$ & $\begin{array}{l}0.315^{* *} \\
(2.155)\end{array}$ & $\begin{array}{l}0.324^{* *} \\
(2.280)\end{array}$ & $\begin{array}{l}0.328^{* *} \\
(2.380)\end{array}$ \\
\hline Log usual hours & $\begin{array}{l}-0.266 * * \\
(2.270)\end{array}$ & $\begin{array}{l}-0.286^{* *} \\
(2.444)\end{array}$ & $\begin{array}{l}-0.442 \\
(1.424)\end{array}$ & $\begin{array}{l}-0.384 \\
(1.231)\end{array}$ & $\begin{array}{l}-0.221 \\
(0.738)\end{array}$ & $\begin{array}{l}-0.172 \\
(0.571)\end{array}$ \\
\hline Job Tenure & $\begin{array}{l}-0.002 * * * \\
(2.964)\end{array}$ & $\begin{array}{l}-0.001 * * \\
(2.013)\end{array}$ & $\begin{array}{l}-0.002 \\
(0.827)\end{array}$ & $\begin{array}{l}-0.001 \\
(0.423)\end{array}$ & $\begin{array}{l}-0.002 \\
(1.225)\end{array}$ & $\begin{array}{l}-0.002 \\
(0.827)\end{array}$ \\
\hline Tenure Squared & $\begin{array}{l}0.000^{* *} \\
(2.171)\end{array}$ & $\begin{array}{l}0.000^{* *} \\
(2.069)\end{array}$ & $\begin{array}{l}0.000 \\
(0.700)\end{array}$ & $\begin{array}{l}0.000 \\
(0.628)\end{array}$ & $\begin{array}{l}0.000 \\
(1.192)\end{array}$ & $\begin{array}{l}0.000 \\
(1.163)\end{array}$ \\
\hline Age & $\begin{array}{l}-0.031^{* *} \\
(2.274)\end{array}$ & & $\begin{array}{l}0.061 \\
(1.631)\end{array}$ & & $\begin{array}{l}0.059 * \\
(1.679)\end{array}$ & \\
\hline Age square & $\begin{array}{l}0.000 * * * \\
(2.655)\end{array}$ & & $\begin{array}{l}-0.001 \\
(1.380)\end{array}$ & & $\begin{array}{l}-0.001 \\
(1.354)\end{array}$ & \\
\hline University degree & $\begin{array}{l}-0.578^{* * *} \\
(6.175)\end{array}$ & & $\begin{array}{l}-0.762 * * * \\
(3.105)\end{array}$ & & $\begin{array}{l}-0.640^{* * *} \\
(2.746)\end{array}$ & \\
\hline $\begin{array}{l}\text { Vocational } \\
\text { qualifications }\end{array}$ & $\begin{array}{l}-0.359 * * * \\
(4.662)\end{array}$ & & $\begin{array}{l}-0.567^{* * *} \\
(3.041)\end{array}$ & & $\begin{array}{l}-0.574^{* * *} \\
(3.232)\end{array}$ & \\
\hline A - levels plus & $\begin{array}{l}-0.337 * * * \\
(3.895)\end{array}$ & & $\begin{array}{l}-0.633^{* * *} \\
(2.900)\end{array}$ & & $\begin{array}{l}-0.569 * * * \\
(2.755)\end{array}$ & \\
\hline $\mathrm{O}$ - levels plus & $\begin{array}{l}-0.234 * * * \\
(2.877)\end{array}$ & & $\begin{array}{l}-0.437^{* *} \\
(2.195)\end{array}$ & & $\begin{array}{l}-0.405^{* *} \\
(2.173)\end{array}$ & \\
\hline $\begin{array}{l}\text { Commercial or } \\
\text { Apprentice }\end{array}$ & $\begin{array}{l}-0.185^{*} \\
(1.850)\end{array}$ & & $\begin{array}{l}-0.178 \\
(0.731)\end{array}$ & & $\begin{array}{l}-0.286 \\
(1.238)\end{array}$ & \\
\hline Married & $\begin{array}{l}0.042 \\
(0.889)\end{array}$ & $\begin{array}{l}0.113^{* * *} \\
(2.589)\end{array}$ & $\begin{array}{l}8 \\
1)\end{array}$ & $\begin{array}{l}0.099 \\
(0.818)\end{array}$ & 556 & $\begin{array}{l}0.126 \\
(1.107)\end{array}$ \\
\hline Mortgage house & $\begin{array}{l}-0.158^{* * * *} \\
(3.098)\end{array}$ & $\begin{array}{l}-0.175^{* * *} \\
(3.445)\end{array}$ & $\begin{array}{l}-0.110 \\
(0.678)\end{array}$ & $\begin{array}{l}-0.186 \\
(1.133)\end{array}$ & $\begin{array}{l}-0.123 \\
(0.796)\end{array}$ & $\begin{array}{l}-0.196 \\
(1.259)\end{array}$ \\
\hline Paid outright house & $\begin{array}{l}-0.056 \\
(0.776)\end{array}$ & $\begin{array}{l}-0.017 \\
(0.234)\end{array}$ & & $\begin{array}{l}-0.069 \\
(0.332)\end{array}$ & $\begin{array}{l}-0.070 \\
(0.360)\end{array}$ & $\begin{array}{l}-0.103 \\
(0.527)\end{array}$ \\
\hline $\begin{array}{l}\text { Promotion } \\
\text { Opportunities }\end{array}$ & $\begin{array}{l}0.370 * * * \\
(9.566)\end{array}$ & $\begin{array}{l}0.346^{* * *} \\
(9.037)\end{array}$ & $\begin{array}{l}0.393 * * * \\
(3.624)\end{array}$ & $\begin{array}{l}0.356^{* * *} \\
(3.269)\end{array}$ & $\begin{array}{l}0.383 * * * \\
(3.760)\end{array}$ & $\begin{array}{l}0.359 * * * \\
(3.518)\end{array}$ \\
\hline Public Sector & $\begin{array}{l}-0.146^{* *} \\
(2.041)\end{array}$ & $\begin{array}{l}-0.151^{* *} \\
(2.101)\end{array}$ & $\begin{array}{l}0.018 \\
(0.099)\end{array}$ & $\begin{array}{l}0.039 \\
(0.209)\end{array}$ & $\begin{array}{l}-0.047 \\
(0.268)\end{array}$ & $\begin{array}{l}-0.039 \\
(0.218)\end{array}$ \\
\hline Works mornings only & $\begin{array}{l}-0.052 \\
(0.390)\end{array}$ & $\begin{array}{l}-0.022 \\
(0.169)\end{array}$ & $\begin{array}{l}0.042 \\
(0.145)\end{array}$ & $\begin{array}{l}0.128 \\
(0.435)\end{array}$ & $\begin{array}{l}0.080 \\
(0.280)\end{array}$ & $\begin{array}{l}0.145 \\
(0.501)\end{array}$ \\
\hline $\begin{array}{l}\text { Works evenings or } \\
\text { nights }\end{array}$ & $\begin{array}{l}-0.246 * * \\
(2.502)\end{array}$ & $\begin{array}{l}-0.240^{* *} \\
(2.443)\end{array}$ & $\begin{array}{l}-0.490^{*} \\
(1.745)\end{array}$ & $\begin{array}{l}-0.464 \\
(1.636)\end{array}$ & $\begin{array}{l}-0.433 \\
(1.594)\end{array}$ & $\begin{array}{l}-0.426 \\
(1.554)\end{array}$ \\
\hline Variable shift patterns & $\begin{array}{l}-0.082 \\
(1.559)\end{array}$ & $\begin{array}{l}-0.076 \\
(1.453)\end{array}$ & $\begin{array}{l}-0.162 \\
(1.164)\end{array}$ & $\begin{array}{l}-0.167 \\
(1.176)\end{array}$ & $\begin{array}{l}-0.146 \\
(1.108)\end{array}$ & $\begin{array}{l}-0.162 \\
(1.211)\end{array}$ \\
\hline Fair health & $\begin{array}{l}0.311 * * * \\
(4.376)\end{array}$ & $\begin{array}{l}0.307^{* * *} \\
(4.305)\end{array}$ & $\begin{array}{l}0.397 * * \\
(2.081)\end{array}$ & $\begin{array}{l}0.368^{*} \\
(1.906)\end{array}$ & $\begin{array}{l}0.324 * \\
(1.744)\end{array}$ & $\begin{array}{l}0.296 \\
(1.579)\end{array}$ \\
\hline Good health & $\begin{array}{l}0.469 * * * \\
(6.648)\end{array}$ & $\begin{array}{l}0.455^{* * *} \\
(6.449)\end{array}$ & $\begin{array}{l}0.562 * * * \\
(3.002)\end{array}$ & $\begin{array}{l}0.503^{* * *} \\
(2.662)\end{array}$ & $\begin{array}{l}0.537 * * * \\
(2.960)\end{array}$ & $\begin{array}{l}0.477^{* * *} * \\
(2.608)\end{array}$ \\
\hline Excellent health & $\begin{array}{l}0.703 * * * \\
(9.272)\end{array}$ & $\begin{array}{l}0.675^{* * *} \\
(8.926)\end{array}$ & $\begin{array}{l}0.884 * * * \\
(4.451)\end{array}$ & $\begin{array}{l}0.780^{* * * *} \\
(3.903)\end{array}$ & $\begin{array}{l}0.823 * * * \\
(4.296)\end{array}$ & $\begin{array}{l}0.725^{* * *} \\
(3.767)\end{array}$ \\
\hline Trade union member & $\begin{array}{l}-0.133^{* * *} \\
(2.719)\end{array}$ & $\begin{array}{l}-0.126^{* *} \\
(2.571)\end{array}$ & $\begin{array}{l}-0.381^{* * *} \\
(3.042)\end{array}$ & $\begin{array}{l}-0.374 * * * \\
(2.947)\end{array}$ & $\begin{array}{l}-0.375^{* * *} \\
(3.163)\end{array}$ & $\begin{array}{l}-0.365^{* * *} \\
(3.047)\end{array}$ \\
\hline Temporary Job & $\begin{array}{l}-0.309^{* * *} \\
(3.565)\end{array}$ & $\begin{array}{l}-0.318^{* * *} \\
(3.654)\end{array}$ & $\begin{array}{l}-0.664^{* * * *} \\
(2.715)\end{array}$ & $\begin{array}{l}-0.638 * * * \\
(2.582)\end{array}$ & $\begin{array}{l}-0.675^{* * *} \\
(2.875)\end{array}$ & $\begin{array}{l}-0.656^{* * *} \\
(2.767)\end{array}$ \\
\hline Incremental pay & $\begin{array}{l}0.219 * * * \\
(5.785)\end{array}$ & $\begin{array}{l}0.212^{* * *} \\
(5.618)\end{array}$ & $\begin{array}{l}0.317 * * * \\
(3.103)\end{array}$ & $\begin{array}{l}0.280^{* * * *} \\
(2.724)\end{array}$ & $\begin{array}{l}0.305 * * * \\
(3.159)\end{array}$ & $\begin{array}{l}0.271^{* * *} \\
(2.789)\end{array}$ \\
\hline Part Time & $\begin{array}{l}-0.206 \\
(1.447)\end{array}$ & $\begin{array}{l}-0.185 \\
(1.296)\end{array}$ & $\begin{array}{l}-0.255 \\
(0.623)\end{array}$ & $\begin{array}{l}-0.268 \\
(0.645)\end{array}$ & $\begin{array}{l}-0.544 \\
(1.395)\end{array}$ & $\begin{array}{l}-0.562 \\
(1.422)\end{array}$ \\
\hline Travel Time & $\begin{array}{l}-0.002^{*} \\
(1.928)\end{array}$ & $\begin{array}{l}-0.002 * * \\
(2.064)\end{array}$ & $\begin{array}{l}-0.008 * * * \\
(2.979)\end{array}$ & $\begin{array}{l}-0.008^{* * *} \\
(2.821)\end{array}$ & $\begin{array}{l}-0.008^{* * *} \\
(3.206)\end{array}$ & $\begin{array}{l}-0.008 * * * \\
(3.123)\end{array}$ \\
\hline Size 25 - & $\begin{array}{l}-0.219 * * * \\
(4.446)\end{array}$ & $\begin{array}{l}-0.213^{* * *} \\
(4.293)\end{array}$ & $\begin{array}{l}-0.186 \\
(1.345)\end{array}$ & $\begin{array}{l}-0.168 \\
(1.193)\end{array}$ & $\begin{array}{l}-0.204 \\
(1.544)\end{array}$ & $\begin{array}{l}-0.187 \\
(1.390)\end{array}$ \\
\hline
\end{tabular}




\begin{tabular}{|c|c|c|c|c|c|c|}
\hline Size 100 - 499 & $\begin{array}{l}-0.272 * * * \\
(5.173)\end{array}$ & $\begin{array}{l}-0.261 * * * \\
(4.904)\end{array}$ & $\begin{array}{l}-0.060 \\
(0.400)\end{array}$ & $\begin{array}{l}-0.083 \\
(0.544)\end{array}$ & $\begin{array}{l}-0.079 \\
(0.552)\end{array}$ & $\begin{array}{l}-0.100 \\
(0.683)\end{array}$ \\
\hline Size 500 plus & $\begin{array}{l}-0.243^{* * *} \\
(4.113)\end{array}$ & $\begin{array}{l}-0.233^{* * *} \\
(3.926)\end{array}$ & $\begin{array}{l}-0.064 \\
(0.381)\end{array}$ & $\begin{array}{l}-0.091 \\
(0.529)\end{array}$ & $\begin{array}{l}-0.121 \\
(0.764)\end{array}$ & $\begin{array}{l}-0.153 \\
(0.943)\end{array}$ \\
\hline Voluntarily Quit & $\begin{array}{l}0.349 * * * \\
(5.880)\end{array}$ & $\begin{array}{l}0.341^{* * *} \\
(5.723)\end{array}$ & $\begin{array}{l}0.319^{*} \\
(1.704)\end{array}$ & $\begin{array}{l}0.328^{*} \\
(1.736)\end{array}$ & $\begin{array}{l}0.316^{*} \\
(1.823)\end{array}$ & $\begin{array}{l}0.324^{*} \\
(1.848)\end{array}$ \\
\hline $\begin{array}{l}\text { Number of weeks not } \\
\text { employed in the last } \\
\text { year }\end{array}$ & $\begin{array}{l}0.008 * * * \\
(3.851)\end{array}$ & $\begin{array}{l}0.007 * * * \\
(3.508)\end{array}$ & $\begin{array}{l}0.016^{* * *} \\
(2.885)\end{array}$ & $\begin{array}{l}0.015^{* * *} \\
(2.608)\end{array}$ & $\begin{array}{l}0.015^{* * *} \\
(2.802)\end{array}$ & $\begin{array}{l}0.014 * * \\
(2.548)\end{array}$ \\
\hline Wave10 & $\begin{array}{l}-0.049 \\
(1.434)\end{array}$ & $\begin{array}{l}-0.055 \\
(1.582)\end{array}$ & $\begin{array}{l}0.068 \\
(0.714)\end{array}$ & $\begin{array}{l}0.078 \\
(0.822)\end{array}$ & $\begin{array}{l}0.102 \\
(1.133)\end{array}$ & $\begin{array}{l}0.111 \\
(1.221)\end{array}$ \\
\hline Wave11 & $\begin{array}{l}-0.059 \\
(1.543)\end{array}$ & $\begin{array}{l}-0.086 * * \\
(2.189)\end{array}$ & $\begin{array}{l}0.028 \\
(0.249)\end{array}$ & $\begin{array}{l}0.031 \\
(0.262)\end{array}$ & $\begin{array}{l}0.046 \\
(0.430)\end{array}$ & $\begin{array}{l}0.049 \\
(0.443)\end{array}$ \\
\hline Rest of England & $\begin{array}{l}-0.236 * * * \\
(4.006)\end{array}$ & $\begin{array}{l}-0.237 * * * \\
(4.016)\end{array}$ & & & & \\
\hline London and South East & $\begin{array}{l}-0.403^{* * *} \\
(5.750)\end{array}$ & $\begin{array}{l}-0.342 * * * \\
(4.795)\end{array}$ & & & & \\
\hline Scotland & $\begin{array}{l}-0.271^{* * *} \\
(4.132)\end{array}$ & $\begin{array}{l}-0.270^{* * *} \\
(4.111)\end{array}$ & & & & \\
\hline $\begin{array}{l}\text { Log real } \\
\text { comparative wage }\end{array}$ & & $\begin{array}{l}-0.569 * * * \\
(3.289)\end{array}$ & & $\begin{array}{l}0.064 \\
(0.135)\end{array}$ & & $\begin{array}{l}0.007 \\
(0.016)\end{array}$ \\
\hline Objective 1 area & & & $\begin{array}{l}0.121 \\
(1.060)\end{array}$ & $\begin{array}{l}0.144 \\
(1.241)\end{array}$ & & \\
\hline Cut 1 & $\begin{array}{l}-5.05 * * * \\
(-10.88) \\
-4.38 * * *\end{array}$ & $\begin{array}{l}-4.63 * * * \\
(-11.07) \\
-3.96 * * *\end{array}$ & $\begin{array}{l}-4.23 * * * \\
(-3.48) \\
-3.57 * * *\end{array}$ & $\begin{array}{l}-4.93 * * * \\
(-4.36) \\
-4.26 * * *\end{array}$ & $\begin{array}{l}-3.62 * * * \\
(-3.11) \\
-2.97 * *\end{array}$ & $\begin{array}{l}-4.35 * * * \\
(-4.02) \\
-3.71 * * *\end{array}$ \\
\hline Cut 2 & $\begin{array}{l}(-9.50) \\
-3.65 * * *\end{array}$ & $\begin{array}{l}(-9.53) \\
-3.22 * * *\end{array}$ & $\begin{array}{l}(-2.96) \\
-2.97 * *\end{array}$ & $\begin{array}{l}(-3.80) \\
-3.66 * * *\end{array}$ & $\begin{array}{l}(-2.57) \\
-2.37 *\end{array}$ & $\begin{array}{l}(-3.45) \\
-3.10^{* * *}\end{array}$ \\
\hline Cut 3 & $\begin{array}{l}(-7.93) \\
-3.12 * * *\end{array}$ & $\begin{array}{l}(-7.79) \\
-2.70 * * *\end{array}$ & $\begin{array}{l}(-2.47) \\
-2.39 *\end{array}$ & $\begin{array}{l}(-3.28) \\
-3.08 * * *\end{array}$ & $\begin{array}{l}(-2.06) \\
-1.79\end{array}$ & $\begin{array}{l}(-2.90) \\
-2.51 * *\end{array}$ \\
\hline Cut 4 & $\begin{array}{l}(-6.80) \\
-2.11 * * *\end{array}$ & $\begin{array}{l}(-6.53) \\
-1.69 * * *\end{array}$ & $\begin{array}{l}(-2.00) \\
-1.31\end{array}$ & $\begin{array}{l}(-2.77) \\
-1.99 *\end{array}$ & $\begin{array}{l}(-1.56) \\
-0.73\end{array}$ & $\begin{array}{l}(-2.37) \\
-1.45\end{array}$ \\
\hline $\begin{array}{l}\text { Cut } 5 \\
\text { Cut } 6\end{array}$ & $\begin{array}{l}(-4.61) \\
0.04 \\
(0.08)\end{array}$ & $\begin{array}{l}(-4.10) \\
0.46 \\
(1.11)\end{array}$ & $\begin{array}{l}(-1.10) \\
0.94 \\
(0.79)\end{array}$ & $\begin{array}{l}(-1.81) \\
0.27 \\
(0.25)\end{array}$ & $\begin{array}{l}(-0.64) \\
1.50 \\
(1.32)\end{array}$ & $\begin{array}{l}(-1.37) \\
0.80 \\
(0.76)\end{array}$ \\
\hline Log Likelihood & -10713 & -10734 & -1477 & -1488 & -1613 & -1624 \\
\hline $\begin{array}{l}\text { Observations } \\
\text { Absolute value of z s }\end{array}$ & $\begin{array}{l}7470 \\
\text { cs in paren }\end{array}$ & $\begin{array}{l}7467 \\
\text { eses }\end{array}$ & 1075 & 1075 & 1177 & 1177 \\
\hline
\end{tabular}




\begin{tabular}{|c|c|c|c|c|c|c|}
\hline & \multicolumn{2}{|c|}{ Scotland } & \multicolumn{2}{|c|}{ London and South } & \multicolumn{2}{|c|}{ Rest of England } \\
\hline & (7) & (8) & $\begin{array}{l}\text { East } \\
(9)\end{array}$ & (10) & (11) & (12) \\
\hline Log usual real gross & $0.215^{* *}$ & 0.152 & $0.220^{*}$ & 0.176 & $0.443^{* * *}$ & $0.373 * * *$ \\
\hline hourly wage & (2.064) & (1.527) & (1.929) & (1.635) & (5.178) & (4.538) \\
\hline Log usual hours & $\begin{array}{l}-0.235 \\
(1.004)\end{array}$ & $\begin{array}{l}-0.288 \\
(1.223)\end{array}$ & $\begin{array}{l}-0.607 * * \\
(2.266)\end{array}$ & $\begin{array}{l}-0.600 * * \\
(2.246)\end{array}$ & $\begin{array}{l}-0.166 \\
(0.853)\end{array}$ & $\begin{array}{l}-0.194 \\
(1.002)\end{array}$ \\
\hline Job Tenure & $\begin{array}{l}-0.002 \\
(1.495)\end{array}$ & $\begin{array}{l}-0.002 \\
(1.260)\end{array}$ & $\begin{array}{l}-0.004^{* *} \\
(2.383)\end{array}$ & $\begin{array}{l}-0.003^{*} \\
(1.901)\end{array}$ & $\begin{array}{l}-0.001 \\
(1.246)\end{array}$ & $\begin{array}{l}-0.001 \\
(0.579)\end{array}$ \\
\hline Tenure Squared & $\begin{array}{l}0.000 \\
(0.656)\end{array}$ & $\begin{array}{l}0.000 \\
(1.108)\end{array}$ & $\begin{array}{l}0.000^{* *} \\
(2.458)\end{array}$ & $\begin{array}{l}0.000^{* *} \\
(2.164)\end{array}$ & $\begin{array}{l}0.000 \\
(0.268)\end{array}$ & $\begin{array}{l}0.000 \\
(0.109)\end{array}$ \\
\hline Age & $\begin{array}{l}-0.109 * * * \\
(3.986)\end{array}$ & & $\begin{array}{l}-0.009 \\
(0.295)\end{array}$ & & $\begin{array}{l}-0.020 \\
(0.922)\end{array}$ & \\
\hline Age square & $\begin{array}{l}0.002 * * * \\
(4.532)\end{array}$ & & $\begin{array}{l}0.000 \\
(0.335)\end{array}$ & & $\begin{array}{l}0.000 \\
(0.918)\end{array}$ & \\
\hline University degree & $\begin{array}{l}-0.142 \\
(0.718)\end{array}$ & & $\begin{array}{l}-0.529 * * \\
(2.513)\end{array}$ & & $\begin{array}{l}-0.795^{* * *} \\
(5.208)\end{array}$ & \\
\hline $\begin{array}{l}\text { Vocational } \\
\text { qualifications }\end{array}$ & $\begin{array}{l}0.058 \\
(0.357)\end{array}$ & & $\begin{array}{l}-0.336^{*} \\
(1.810)\end{array}$ & & $\begin{array}{l}-0.487 * * * \\
(3.904)\end{array}$ & \\
\hline A - levels plus & $\begin{array}{l}0.067 \\
(0.388)\end{array}$ & & $\begin{array}{l}-0.154 \\
(0.743)\end{array}$ & & $\begin{array}{l}-0.450^{* * *} \\
(3.151)\end{array}$ & \\
\hline $\mathrm{O}$ - levels plus & $\begin{array}{l}0.072 \\
(0.435)\end{array}$ & & $\begin{array}{l}-0.420 * * \\
(2.079)\end{array}$ & & $\begin{array}{l}-0.275^{* *} \\
(2.071)\end{array}$ & \\
\hline $\begin{array}{l}\text { Commercial or } \\
\text { Apprentice }\end{array}$ & $\begin{array}{l}0.111 \\
(0.442)\end{array}$ & & $\begin{array}{l}-0.072 \\
(0.292)\end{array}$ & & $\begin{array}{l}-0.391 * * \\
(2.564)\end{array}$ & \\
\hline Married & $\begin{array}{l}0.153 \\
(1.534)\end{array}$ & $\begin{array}{l}0.208^{* *} \\
(2.233)\end{array}$ & $\begin{array}{l}-0.177^{*} \\
(1.669)\end{array}$ & $\begin{array}{l}-0.122 \\
(1.281)\end{array}$ & $\begin{array}{l}0.119 \\
(1.570)\end{array}$ & $\begin{array}{l}0.171^{* *} \\
(2.433)\end{array}$ \\
\hline Mortgage house & $\begin{array}{l}-0.150 \\
(1.457)\end{array}$ & $\begin{array}{l}-0.131 \\
(1.259)\end{array}$ & $\begin{array}{l}0.193^{*} \\
(1.803)\end{array}$ & $\begin{array}{l}0.179^{*} \\
(1.681)\end{array}$ & $\begin{array}{l}-0.346^{* * *} \\
(4.219)\end{array}$ & $\begin{array}{l}-0.369^{* * *} \\
(4.528)\end{array}$ \\
\hline Paid outright house & $\begin{array}{l}0.003 \\
(0.015)\end{array}$ & $\begin{array}{l}0.166 \\
(1.003)\end{array}$ & $\begin{array}{l}0.372^{* * *} \\
(2.488)\end{array}$ & $\begin{array}{l}0.387^{* * * *} \\
(2.638)\end{array}$ & $\begin{array}{l}-0.225^{* *} \\
(1.967)\end{array}$ & $\begin{array}{l}-0.230 * * \\
(2.057)\end{array}$ \\
\hline Promotion & $0.335 * * *$ & $0.271^{* * *}$ & $0.309 * * *$ & $0.309 * * *$ & $\begin{array}{l}0.426 * * * \\
(7098)\end{array}$ & $\begin{array}{l}0.408^{* * * *} \\
(6.852)\end{array}$ \\
\hline $\begin{array}{l}\text { Opportunities } \\
\text { Public Sector }\end{array}$ & $\begin{array}{l}(3.949) \\
-0.179 \\
(1.238)\end{array}$ & $\begin{array}{l}(3.231) \\
-0.157 \\
(1.073)\end{array}$ & $\begin{array}{l}(3.647) \\
-0.154 \\
(1.001)\end{array}$ & $\begin{array}{l}(3.679) \\
-0.163 \\
(1.061)\end{array}$ & $\begin{array}{l}(7.098) \\
-0.156 \\
(1.269)\end{array}$ & $\begin{array}{l}(6.852) \\
-0.163 \\
(1.317)\end{array}$ \\
\hline Works mornings only & $\begin{array}{l}-0.091 \\
(0.272)\end{array}$ & $\begin{array}{l}-0.171 \\
(0.503)\end{array}$ & $\begin{array}{l}-0.512^{*} \\
(1.825)\end{array}$ & $\begin{array}{l}-0.470^{*} \\
(1.677)\end{array}$ & $\begin{array}{l}0.163 \\
(0.749)\end{array}$ & $\begin{array}{l}0.195 \\
(0.894)\end{array}$ \\
\hline $\begin{array}{l}\text { Works evenings or } \\
\text { nights }\end{array}$ & $\begin{array}{l}-0.104 \\
(0.528)\end{array}$ & $\begin{array}{l}-0.123 \\
(0.620)\end{array}$ & $\begin{array}{l}-0.199 \\
(0.817)\end{array}$ & $\begin{array}{l}-0.200 \\
(0.824)\end{array}$ & $\begin{array}{l}-0.285^{*} \\
(1.909)\end{array}$ & $\begin{array}{l}-0.249 * \\
(1.662)\end{array}$ \\
\hline Variable shift patterns & $\begin{array}{l}-0.025 \\
(0.224)\end{array}$ & $\begin{array}{l}-0.041 \\
(0.371)\end{array}$ & $\begin{array}{l}-0.099 \\
(0.759)\end{array}$ & $\begin{array}{l}-0.092 \\
(0.705)\end{array}$ & $\begin{array}{l}-0.082 \\
(1.007)\end{array}$ & $\begin{array}{l}-0.070 \\
(0.860)\end{array}$ \\
\hline Fair health & $\begin{array}{l}0.310^{*} \\
(1.847)\end{array}$ & $\begin{array}{l}0.296^{*} \\
(1.750)\end{array}$ & $\begin{array}{l}0.394 * * \\
(2.533)\end{array}$ & $\begin{array}{l}0.410^{* * *} \\
(2.630)\end{array}$ & $\begin{array}{l}0.283 * * * \\
(2.642)\end{array}$ & $\begin{array}{l}0.277^{* * *} \\
(2.579)\end{array}$ \\
\hline Good health & $\begin{array}{l}0.363^{* *} \\
(2.197)\end{array}$ & $\begin{array}{l}0.371^{* *} \\
(2.240)\end{array}$ & $\begin{array}{l}0.618^{* * *} \\
(4.009)\end{array}$ & $\begin{array}{l}0.611^{* * *} \\
(3.965)\end{array}$ & $\begin{array}{l}0.427 * * * \\
(3.966)\end{array}$ & $\begin{array}{l}0.416^{* * *} \\
(3.861)\end{array}$ \\
\hline Excellent health & $\begin{array}{l}0.679 * * * \\
(3.886)\end{array}$ & $\begin{array}{l}0.657 * * * \\
(3.754)\end{array}$ & $\begin{array}{l}0.892^{* * *} \\
(5.383)\end{array}$ & $\begin{array}{l}0.891^{* * * *} \\
(5.379)\end{array}$ & $\begin{array}{l}0.598^{* * * *} \\
(5.103)\end{array}$ & $\begin{array}{l}0.577^{* * *} \\
(4.932)\end{array}$ \\
\hline Trade union member & $\begin{array}{l}-0.104 \\
(1.023)\end{array}$ & $\begin{array}{l}-0.080 \\
(0.784)\end{array}$ & $\begin{array}{l}-0.137 \\
(1.154)\end{array}$ & $\begin{array}{l}-0.110 \\
(0.933)\end{array}$ & $\begin{array}{l}-0.071 \\
(0.897)\end{array}$ & $\begin{array}{l}-0.063 \\
(0.808)\end{array}$ \\
\hline Temporary Job & $\begin{array}{l}-0.474^{* * *} \\
(2.626)\end{array}$ & $\begin{array}{l}-0.414^{* *} \\
(2.288)\end{array}$ & $\begin{array}{l}-0.427^{* *} \\
(2.316)\end{array}$ & $\begin{array}{l}-0.430 * * \\
(2.342)\end{array}$ & $\begin{array}{l}-0.094 \\
(0.666)\end{array}$ & $\begin{array}{l}-0.120 \\
(0.846)\end{array}$ \\
\hline Incremental pay & $\begin{array}{l}0.292 * * * \\
(3.594)\end{array}$ & $\begin{array}{l}0.299 * * * \\
(3.656)\end{array}$ & $\begin{array}{l}0.188 * * \\
(2.248)\end{array}$ & $\begin{array}{l}0.179 * * \\
(2.143)\end{array}$ & $\begin{array}{l}0.161 * * * \\
(2.720)\end{array}$ & $\begin{array}{l}0.162 * * * \\
(2.736)\end{array}$ \\
\hline Part Time & $\begin{array}{l}-0.270 \\
(0.941)\end{array}$ & $\begin{array}{l}-0.319 \\
(1.097)\end{array}$ & $\begin{array}{l}0.227 \\
(0.707)\end{array}$ & $\begin{array}{l}0.232 \\
(0.727)\end{array}$ & $\begin{array}{l}-0.227 \\
(1.008)\end{array}$ & $\begin{array}{l}-0.160 \\
(0.706)\end{array}$ \\
\hline Travel Time & $\begin{array}{l}-0.004 * \\
(1.875)\end{array}$ & $\begin{array}{l}-0.004 * \\
(1.796)\end{array}$ & $\begin{array}{l}0.000 \\
(0.247)\end{array}$ & $\begin{array}{l}0.000 \\
(0.113)\end{array}$ & $\begin{array}{l}-0.001 \\
(0.799)\end{array}$ & $\begin{array}{l}-0.001 \\
(0.927)\end{array}$ \\
\hline
\end{tabular}




\begin{tabular}{|c|c|c|c|c|c|c|}
\hline Size 25 - 99 & $\begin{array}{l}-0.151 \\
(1.410)\end{array}$ & $\begin{array}{l}-0.144 \\
(1.337)\end{array}$ & $\begin{array}{l}-0.013 \\
(0.116)\end{array}$ & $\begin{array}{l}0.005 \\
(0.043)\end{array}$ & $\begin{array}{l}-0.330 * * * \\
(4.305)\end{array}$ & $\begin{array}{l}-0.334 * * * \\
(4.318)\end{array}$ \\
\hline Size 100 - 499 & $\begin{array}{l}-0.366^{* * *} \\
(3.152)\end{array}$ & $\begin{array}{l}-0.369 * * * \\
(3.121)\end{array}$ & $\begin{array}{l}-0.166 \\
(1.457)\end{array}$ & $\begin{array}{l}-0.143 \\
(1.236)\end{array}$ & $\begin{array}{l}-0.335^{* * *} \\
(4.107)\end{array}$ & $\begin{array}{l}-0.326 * * * \\
(3.931)\end{array}$ \\
\hline Size 500 plus & $\begin{array}{l}-0.252 * * \\
(2.085)\end{array}$ & $\begin{array}{l}-0.214^{*} \\
(1.766)\end{array}$ & $\begin{array}{l}-0.252^{* *} \\
(1.990)\end{array}$ & $\begin{array}{l}-0.231^{*} \\
(1.812)\end{array}$ & $\begin{array}{l}-0.268 * * * \\
(2.767)\end{array}$ & $\begin{array}{l}-0.251^{* *} \\
(2.575)\end{array}$ \\
\hline Voluntarily Quit & $\begin{array}{l}0.265^{*} \\
(1.937)\end{array}$ & $\begin{array}{l}0.237^{*} \\
(1.719)\end{array}$ & $\begin{array}{l}0.419 * * * \\
(3.493)\end{array}$ & $\begin{array}{l}0.421^{* * *} \\
(3.511)\end{array}$ & $\begin{array}{l}0.336 * * * \\
(3.661)\end{array}$ & $\begin{array}{l}0.328 * * * \\
(3.579)\end{array}$ \\
\hline $\begin{array}{l}\text { Number of weeks not } \\
\text { employed in the last } \\
\text { year }\end{array}$ & $\begin{array}{l}0.007^{*} \\
(1.710)\end{array}$ & $\begin{array}{l}0.007^{*} \\
(1.718)\end{array}$ & $\begin{array}{l}0.009 * * \\
(2.052)\end{array}$ & $\begin{array}{l}0.008 * * \\
(2.001)\end{array}$ & $\begin{array}{l}0.004 \\
(1.070)\end{array}$ & $\begin{array}{l}0.003 \\
(0.833)\end{array}$ \\
\hline Wave10 & $\begin{array}{l}0.004 \\
(0.051)\end{array}$ & $\begin{array}{l}0.006 \\
(0.080)\end{array}$ & $\begin{array}{l}-0.062 \\
(0.817)\end{array}$ & $\begin{array}{l}-0.089 \\
(1.145)\end{array}$ & $\begin{array}{l}-0.116^{* *} \\
(2.214)\end{array}$ & $\begin{array}{l}-0.125 * * \\
(2.378)\end{array}$ \\
\hline Wave11 & $\begin{array}{l}-0.101 \\
(1.103)\end{array}$ & $\begin{array}{l}-0.116 \\
(1.231)\end{array}$ & $\begin{array}{l}-0.058 \\
(0.730)\end{array}$ & $\begin{array}{l}-0.105 \\
(1.259)\end{array}$ & $\begin{array}{l}-0.104^{*} \\
(1.814)\end{array}$ & $\begin{array}{l}-0.137 * * \\
(2.336)\end{array}$ \\
\hline $\begin{array}{l}\text { Log real } \\
\text { comparative wage }\end{array}$ & & $\begin{array}{l}-0.476 \\
(1.188)\end{array}$ & & $\begin{array}{l}-0.683^{*} \\
(1.771)\end{array}$ & & $\begin{array}{l}-0.675 * * \\
(2.547)\end{array}$ \\
\hline Cut 1 & $\begin{array}{l}-5.66 * * * \\
(-5.94)\end{array}$ & $\begin{array}{l}-4.36 * * * \\
(-5.11)\end{array}$ & $\begin{array}{l}-5.52 * * * \\
(-5.18)\end{array}$ & $\begin{array}{l}-5.37 * * * \\
(-5.59)\end{array}$ & $\begin{array}{l}-4.56 * * * \\
(-6.02)\end{array}$ & $\begin{array}{l}-4.15 * * * \\
(-6.08)\end{array}$ \\
\hline Cut 2 & $\begin{array}{l}-4.83 * * * \\
(-5.12)\end{array}$ & $\begin{array}{l}-3.52 * * * \\
(-4.18)\end{array}$ & $\begin{array}{l}-4.78 * * * \\
(-4.51)\end{array}$ & $\begin{array}{l}-4.63 * * * \\
(-4.85)\end{array}$ & $\begin{array}{l}-3.98^{* * *} \\
(-5.26)\end{array}$ & $\begin{array}{l}-3.57 * * * \\
(-5.24)\end{array}$ \\
\hline Cut 3 & $\begin{array}{l}-4.14 * * * \\
(-4.43)\end{array}$ & $\begin{array}{l}-2.84 * * * \\
(-3.40)\end{array}$ & $\begin{array}{l}-3.96 * * * \\
(-3.75)\end{array}$ & $\begin{array}{l}-3.81 * * * \\
(-4.01)\end{array}$ & $\begin{array}{l}-3.19 * * * \\
(-4.23)\end{array}$ & $\begin{array}{l}-2.78 * * * \\
(-4.09)\end{array}$ \\
\hline Cut 4 & $\begin{array}{l}-3.60 * * * \\
(-3.87)\end{array}$ & $\begin{array}{l}-2.30 * * * \\
(-2.76)\end{array}$ & $\begin{array}{l}-3.44 * * * \\
(-3.27)\end{array}$ & $\begin{array}{l}-3.30 * * * \\
(-3.48)\end{array}$ & $\begin{array}{l}-2.66^{* * * *} \\
(-3.53)\end{array}$ & $\begin{array}{l}-2.25 * * * \\
(-3.31)\end{array}$ \\
\hline Cut 5 & $\begin{array}{l}-2.69 * * * \\
(-2.90)\end{array}$ & $\begin{array}{l}-1.39 * \\
(-1.67)\end{array}$ & $\begin{array}{l}-2.42 * * \\
(-2.31)\end{array}$ & $\begin{array}{l}-2.28 * * \\
(-2.42)\end{array}$ & $\begin{array}{l}-1.59 * * \\
(-2.11)\end{array}$ & $\begin{array}{l}-1.18^{*} \\
(-1.73)\end{array}$ \\
\hline Cut 6 & $\begin{array}{l}-0.59 \\
(-0.64)\end{array}$ & $\begin{array}{l}0.71 \\
(0.86)\end{array}$ & $\begin{array}{l}-0.14 \\
(-0.13)\end{array}$ & $\begin{array}{l}0.01 \\
(0.01)\end{array}$ & $\begin{array}{l}0.56 \\
(0.74)\end{array}$ & $\begin{array}{l}0.96 \\
(1.42)\end{array}$ \\
\hline Log Likelihood & -2234 & -2248 & -2171 & -2175 & -4572 & -4584 \\
\hline $\begin{array}{l}\text { Observations } \\
\text { Absolute value }\end{array}$ & 1529 & 1528 & 1562 & 1561 & 3202 & 3201 \\
\hline
\end{tabular}


Table 3C: OVERALL JOB SATISFACTION - FEMALES

National

\begin{tabular}{|c|c|c|c|c|c|c|}
\hline & & & objective & variable & objecti & I variable \\
\hline & (1) & (2) & (3) & $(4)$ & (5) & (6) \\
\hline Log usual real gross & $0.078^{*}$ & 0.026 & 0.072 & -0.003 & 0.044 & -0.020 \\
\hline hourly wage & $(1.651)$ & $(0.563)$ & $(0.554)$ & $(0.027)$ & $(0.355)$ & $(0.163)$ \\
\hline Log usual hours & $-0.162 * * *$ & $-0.170 * * *$ & 0.042 & 0.021 & 0.037 & 0.024 \\
\hline & $(2.860)$ & (2.989) & $(0.291)$ & $(0.147)$ & $(0.270)$ & $(0.181)$ \\
\hline Job Tenure & $-0.002 * * *$ & $-0.002 * *$ & -0.003 & -0.002 & $-0.004 * *$ & $-0.003 *$ \\
\hline & & & & & & \\
\hline Tenure Squared & $0.000 * *$ & $0.000 * *$ & 0.000 & 0.000 & $0.000 *$ & $0.000 *$ \\
\hline & $(2.138)$ & $(2.095)$ & $(1.021)$ & $(1.074)$ & (1.789) & (1.830) \\
\hline Age & $-0.023 *$ & & -0.043 & & -0.037 & \\
\hline & $(1.861)$ & & $(1.285)$ & & $(1.160)$ & \\
\hline Age square & $0.000 * *$ & & $0.001 *$ & & $0.001 *$ & \\
\hline & $(2.504)$ & & (1.829) & & (1.699) & \\
\hline University degree & $-0.519 * * *$ & & $-0.522 * *$ & & $-0.411^{* *}$ & \\
\hline & $(6.063)$ & & $(2.332)$ & & $(1.972)$ & \\
\hline Vocational & $-0.301 * * *$ & & $-0.303 *$ & & -0.131 & \\
\hline qualifications & $(4.282)$ & & $(1.662)$ & & $(0.791)$ & \\
\hline A - levels plus & $-0.255^{* * *}$ & & $-0.351 *$ & & -0.242 & \\
\hline & $(3.306)$ & & (1.814) & & $(1.360)$ & \\
\hline O - levels plus & $-0.185^{* * *}$ & & -0.207 & & -0.107 & \\
\hline & $(2.655)$ & & (1.166) & & $(0.664)$ & \\
\hline Commercial or & $-0.177 * *$ & & -0.177 & & -0.202 & \\
\hline Apprentice & $(2.025)$ & & $(0.850)$ & & $(1.076)$ & \\
\hline Married & $0.169 * * *$ & $0.242 * * *$ & $0.273 * *$ & $0.372 * * *$ & $0.276^{* * *}$ & $0.363 * * *$ \\
\hline & $(4.162)$ & $(6.281)$ & $(2.544)$ & $(3.666)$ & $(2.722)$ & $(3.774)$ \\
\hline Mortgage house & -0.071 & $-0.085 *$ & 0.009 & -0.060 & -0.009 & -0.065 \\
\hline & (1.589) & $(1.904)$ & $(0.067)$ & $(0.434)$ & $(0.066)$ & $(0.500)$ \\
\hline Paid outright house & -0.090 & -0.029 & -0.007 & 0.044 & 0.034 & 0.083 \\
\hline & $(1.372)$ & $(0.452)$ & $(0.041)$ & $(0.258)$ & $(0.210)$ & $(0.511)$ \\
\hline Promotion & $0.233^{* * *}$ & $0.205^{* * *}$ & $0.179 *$ & 0.128 & $0.191 * *$ & $0.149 *$ \\
\hline Opportunities & $(6.720)$ & $(5.955)$ & $(1.856)$ & $(1.340)$ & $(2.111)$ & (1.666) \\
\hline Public Sector & 0.018 & 0.025 & 0.125 & 0.114 & 0.091 & 0.084 \\
\hline & $(0.317)$ & $(0.445)$ & $(0.776)$ & $(0.702)$ & $(0.591)$ & $(0.542)$ \\
\hline Works mornings only & $0.145^{* *}$ & $0.174 * *$ & 0.219 & 0.281 & 0.213 & 0.259 \\
\hline & $(2.070)$ & $(2.492)$ & $(1.231)$ & $(1.592)$ & $(1.256)$ & $(1.540)$ \\
\hline Works evenings or & -0.067 & -0.071 & 0.171 & 0.109 & 0.094 & 0.054 \\
\hline nights & $(0.888)$ & $(0.942)$ & $(0.906)$ & $(0.580)$ & $(0.528)$ & $(0.304)$ \\
\hline Variable shift patterns & 0.012 & 0.027 & $0.309 * *$ & $0.301 * *$ & 0.188 & 0.182 \\
\hline & $(0.222)$ & $(0.484)$ & $(2.194)$ & $(2.156)$ & $(1.430)$ & $(1.392)$ \\
\hline Fair health & -0.028 & -0.028 & -0.134 & -0.136 & -0.089 & -0.092 \\
\hline & $(0.453)$ & $(0.451)$ & $(0.786)$ & $(0.801)$ & $(0.552)$ & $(0.573)$ \\
\hline Good health & $0.167 * * *$ & $0.153 * *$ & 0.081 & 0.052 & 0.125 & 0.101 \\
\hline & $(2.766)$ & $(2.537)$ & $(0.497)$ & $(0.320)$ & $(0.814)$ & $(0.662)$ \\
\hline Excellent health & $0.382 * * *$ & $0.362 * * *$ & 0.193 & 0.154 & 0.262 & 0.237 \\
\hline & $(5.789)$ & $(5.482)$ & $(1.120)$ & $(0.900)$ & (1.606) & $(1.462)$ \\
\hline Trade union member & $-0.259 * * *$ & $-0.259 * * *$ & $-0.312 * * *$ & $-0.322 * * *$ & $-0.217 * *$ & $-0.223^{* *}$ \\
\hline & $(5.993)$ & $(5.990)$ & $(2.797)$ & $(2.904)$ & $(2.063)$ & $(2.131)$ \\
\hline Temporary Job & $-0.226 * * *$ & $-0.242 * * *$ & -0.259 & $-0.306^{*}$ & -0.264 & $-0.302 *$ \\
\hline & $(3.408)$ & (3.672) & $(1.453)$ & $(1.723)$ & $(1.529)$ & $(1.760)$ \\
\hline Incremental pay & $0.194 * * *$ & $0.184 * * *$ & $0.334 * * *$ & $0.314 * * *$ & $0.307 * * *$ & $0.283^{* * *}$ \\
\hline & $(5.578)$ & $(5.300)$ & (3.359) & $(3.180)$ & (3.294) & $(3.056)$ \\
\hline
\end{tabular}




\begin{tabular}{|c|c|c|c|c|c|c|}
\hline Part Time & $\begin{array}{l}-0.109 * \\
(1.920)\end{array}$ & $\begin{array}{l}-0.091 \\
(1.566)\end{array}$ & $\begin{array}{l}-0.130 \\
(0.885)\end{array}$ & $\begin{array}{l}-0.121 \\
(0.815)\end{array}$ & $\begin{array}{l}-0.207 \\
(1.491)\end{array}$ & $\begin{array}{l}-0.207 \\
(1.469)\end{array}$ \\
\hline Travel Time & $\begin{array}{l}-0.002 \\
(1.525)\end{array}$ & $\begin{array}{l}-0.002^{* *} \\
(1.961)\end{array}$ & $\begin{array}{l}0.001 \\
(0.411)\end{array}$ & $\begin{array}{l}0.001 \\
(0.160)\end{array}$ & $\begin{array}{l}0.001 \\
(0.462)\end{array}$ & $\begin{array}{l}0.001 \\
(0.356)\end{array}$ \\
\hline Size 25 - 99 & $\begin{array}{l}-0.111^{* * *} \\
(2.652)\end{array}$ & $\begin{array}{l}-0.097^{* *} \\
(2.308)\end{array}$ & $\begin{array}{l}-0.202 * \\
(1.738)\end{array}$ & $\begin{array}{l}-0.185 \\
(1.595)\end{array}$ & $\begin{array}{l}-0.199 * \\
(1.830)\end{array}$ & $\begin{array}{l}-0.198 * \\
(1.823)\end{array}$ \\
\hline Size 100 - 499 & $\begin{array}{l}-0.187 * * * \\
(3.924)\end{array}$ & $\begin{array}{l}-0.170 * * * \\
(3.525)\end{array}$ & $\begin{array}{l}-0.101 \\
(0.737)\end{array}$ & $\begin{array}{l}-0.090 \\
(0.648)\end{array}$ & $\begin{array}{l}-0.126 \\
(0.977)\end{array}$ & $\begin{array}{l}-0.128 \\
(0.984)\end{array}$ \\
\hline Size 500 plus & $\begin{array}{l}-0.197 * * * \\
(3.788)\end{array}$ & $\begin{array}{l}-0.180 * * * \\
(3.405)\end{array}$ & $\begin{array}{l}-0.381 * * * \\
(2.610)\end{array}$ & $\begin{array}{l}-0.361 * * \\
(2.437)\end{array}$ & $\begin{array}{l}-0.324 * * \\
(2.345)\end{array}$ & $\begin{array}{l}-0.319 * * \\
(2.268)\end{array}$ \\
\hline Voluntarily Quit & $\begin{array}{l}0.300^{* * *} \\
(5.487)\end{array}$ & $\begin{array}{l}0.295^{* * *} \\
(5.397)\end{array}$ & $\begin{array}{l}0.225 \\
(1.313)\end{array}$ & $\begin{array}{l}0.210 \\
(1.224)\end{array}$ & $\begin{array}{l}0.190 \\
(1.193)\end{array}$ & $\begin{array}{l}0.172 \\
(1.081)\end{array}$ \\
\hline $\begin{array}{l}\text { Number of weeks not } \\
\text { employed in the last } \\
\text { year }\end{array}$ & $\begin{array}{l}0.003 * * \\
(2.092)\end{array}$ & $\begin{array}{l}0.002 \\
(1.475)\end{array}$ & $\begin{array}{l}0.006 \\
(1.385)\end{array}$ & $\begin{array}{l}0.005 \\
(1.154)\end{array}$ & $\begin{array}{l}0.005 \\
(1.164)\end{array}$ & $\begin{array}{l}0.004 \\
(0.940)\end{array}$ \\
\hline Wave 10 & $\begin{array}{l}-0.118^{* * *} \\
(3.682)\end{array}$ & $\begin{array}{l}-0.108^{* * *} \\
(3.381)\end{array}$ & $\begin{array}{l}0.056 \\
(0.633)\end{array}$ & $\begin{array}{l}0.065 \\
(0.736)\end{array}$ & $\begin{array}{l}0.031 \\
(0.373)\end{array}$ & $\begin{array}{l}0.040 \\
(0.480)\end{array}$ \\
\hline Wave 11 & $\begin{array}{l}-0.034 \\
(0.966)\end{array}$ & $\begin{array}{l}0.022 \\
(0.537)\end{array}$ & $\begin{array}{l}0.158 \\
(1.557)\end{array}$ & $\begin{array}{l}0.206^{*} \\
(1.747)\end{array}$ & $\begin{array}{l}0.124 \\
(1.303)\end{array}$ & $\begin{array}{l}0.149 \\
(1.346)\end{array}$ \\
\hline Rest of England & $\begin{array}{l}-0.117 * * \\
(2.224)\end{array}$ & $\begin{array}{l}-0.127 * * \\
(2.398)\end{array}$ & & & & \\
\hline London and South East & $\begin{array}{l}-0.205^{* * *} \\
(3.351)\end{array}$ & $\begin{array}{l}-0.169 * * * \\
(2.691)\end{array}$ & & & & \\
\hline Scotland & $\begin{array}{l}-0.158 * * * \\
(2.740)\end{array}$ & $\begin{array}{l}-0.172 * * * \\
(2.982)\end{array}$ & & & & \\
\hline $\begin{array}{l}\text { Log real } \\
\text { comparative wage }\end{array}$ & & $\begin{array}{l}-0.393^{* *} \\
(2.475)\end{array}$ & & $\begin{array}{l}-0.307 \\
(0.709)\end{array}$ & & $\begin{array}{l}-0.086 \\
(0.212)\end{array}$ \\
\hline Objective 1 area & & & $\begin{array}{l}0.164^{*} \\
(1.700)\end{array}$ & $\begin{array}{l}0.143 \\
(1.493)\end{array}$ & & \\
\hline Cut 1 & $\begin{array}{l}-4.16^{* * *} \\
(-10.98)\end{array}$ & $\begin{array}{l}-3.83^{* * *} \\
(-12.48)\end{array}$ & $\begin{array}{l}-5.48 * * * \\
(-4.77)\end{array}$ & $\begin{array}{l}-4.96 * * * \\
(-5.09)\end{array}$ & $\begin{array}{l}-5.38 * * * \\
(-4.89)\end{array}$ & $\begin{array}{l}-4.92 * * * \\
(-5.24)\end{array}$ \\
\hline Cut 2 & $\begin{array}{l}-3.53 * * * \\
(-9.37)\end{array}$ & $\begin{array}{l}-3.20 * * * \\
(-10.51)\end{array}$ & $\begin{array}{l}-4.77 * * * \\
(-4.20)\end{array}$ & $\begin{array}{l}-4.26 * * * \\
(-4.43)\end{array}$ & $\begin{array}{l}-4.69 * * * \\
(-4.31)\end{array}$ & $\begin{array}{l}-4.25 * * * \\
(-4.58)\end{array}$ \\
\hline Cut 3 & $\begin{array}{l}-2.85 * * * \\
(-7.61)\end{array}$ & $\begin{array}{l}-2.52 * * * \\
(-8.34)\end{array}$ & $\begin{array}{l}-4.07 * * * \\
(-3.60)\end{array}$ & $\begin{array}{l}-3.57 * * * \\
(-3.75)\end{array}$ & $\begin{array}{l}-3.98 * * * \\
(-3.68)\end{array}$ & $\begin{array}{l}-3.55 * * * \\
(-3.85)\end{array}$ \\
\hline Cut 4 & $\begin{array}{l}-2.49 * * * \\
(-6.65)\end{array}$ & $\begin{array}{l}-2.16^{* * *} \\
(-7.16)\end{array}$ & $\begin{array}{l}-3.74 * * * \\
(-3.32)\end{array}$ & $\begin{array}{l}-3.26 * * * \\
(-3.42)\end{array}$ & $\begin{array}{l}-3.65 * * * \\
(-3.38)\end{array}$ & $\begin{array}{l}-3.22 * * * \\
(-3.51)\end{array}$ \\
\hline Cut 5 & $\begin{array}{l}-1.58 * * * \\
(-4.23)\end{array}$ & $\begin{array}{l}-1.25^{* * *} \\
(-4.16)\end{array}$ & $\begin{array}{l}-2.84 * * \\
(-2.54)\end{array}$ & $\begin{array}{l}-2.37 * * \\
(-2.50)\end{array}$ & $\begin{array}{l}-2.78 * * \\
(-2.59)\end{array}$ & $\begin{array}{l}-2.36 * * \\
(-2.58)\end{array}$ \\
\hline Cut 6 & $\begin{array}{l}0.44 \\
(1.18)\end{array}$ & $\begin{array}{l}0.76 * * \\
(2.52)\end{array}$ & $\begin{array}{l}-0.85 \\
(-0.77)\end{array}$ & $\begin{array}{l}-0.40 \\
(-0.43)\end{array}$ & $\begin{array}{l}-0.85 \\
(-0.80)\end{array}$ & $\begin{array}{l}-0.45 \\
(-0.49)\end{array}$ \\
\hline Log Likelihood & -11542 & -11573 & -1531 & -1542 & -1678 & -1688 \\
\hline Observations & 8446 & 8444 & 1155 & 1154 & 1252 & 1251 \\
\hline
\end{tabular}


Scotland

Log usual real gross
hourly wage
Log usual hours
Job Tenure

Tenure Squared

Age

Age square

University degree

Vocational

qualifications

A - levels plus

$\mathrm{O}$ - levels plus

Commercial or

Apprentice

Married

Mortgage house

Paid outright house

Promotion

Opportunities

Public Sector

Works mornings only

Works evenings or

nights

Variable shift patterns

Fair health

Good health

Excellent health

Trade union member

Temporary Job

Incremental pay

Part Time

Travel Time

\section{(7)}

(0.501)

0.086

$(0.627)$

$-0.003$

(1.582)

0.000

(1.183)

$-0.028$

(0.997)

0.000

(1.283)

-0.404 **

(2.265)

$-0.364 * *$

(2.399)

$-0.232$

(1.449)

$-0.131$

(0.877)

$-0.276$

(1.303)

0.060

(0.677)

$-0.117$

(1.239)

0.097

(0.624)

$0.168^{* *}$

(2.198)

0.163

(1.292)

$0.336^{* *}$

(1.999)

0.044

$(0.266)$

0.004

$(0.033)$

$-0.171$

(1.172)

0.047

(0.337)

$0.350^{* *}$

(2.343)

$-0.347 * * *$

(3.717)

$-0.209$

(1.370)

$0.215^{* * *}$

(2.770)

$-0.411 * * *$

(3.226)

$-0.004 * *$

(1.991)

(8)

0.013

(0.124)

0.084

(0.611)

$-0.002$

(1.118)

0.000

(1.301)

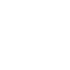

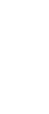

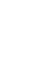

0.151*

(1.822)

$-0.125$

0.169

(1.103)

$0.128^{*}$

(1.695)

0.180

(1.429)

(2.163)

0.050

(0.303)

0.005

$(0.045)$

$-0.181$

(1.242)

0.033

$(0.237)$

$-0.211$

(1.385)

(2.758)
0.053

(1.301)

East

\begin{tabular}{ll}
\multicolumn{1}{c}{$(9)$} & \multicolumn{1}{c}{$(10)$} \\
0.113 & 0.076 \\
$(1.214)$ & $(0.847)$ \\
-0.118 & -0.104 \\
$(0.990)$ & $(0.874)$ \\
-0.002 & -0.001 \\
$(1.497)$ & $(0.914)$ \\
0.000 & 0.000 \\
$(1.480)$ & $(1.226)$ \\
0.005 & \\
$(0.196)$ & \\
-0.000 & \\
$(0.066)$ & \\
-0.745 &
\end{tabular}

$-0.745^{* * *}$

(3.835)

$-0.595^{* * *}$

(3.526)

-0.431 **

(2.284)

$-0.582^{* * *}$

(3.376)

$-0.487 * *$

(2.363)

(1.325)

$0.363^{* *}$

$0.329 * *$

(2.207)

$-0.333^{* * *}$

(3.578)

$0.203^{* * *}$

(2.619)

$-0.360^{* * *}$

$-0.005^{* *}$

(2.150)

(3.299) (3.038)

$-0.306 * * * \quad-0.321 * * *$

(0.540)

$0.283 * * *$

$(0.711)$

$0.280^{* * *}$

$-0.096$

$(0.747)$

$-0.118$

$(0.900)$

$-0.001$

(0.449)

(0.573)
Rest of England

(11)

$-0.384 * * * \quad-0.407 *$

(4.269) (4.514)

$-0.002 * \quad-0.001$

(1.797) (0.978)

$0.000 \quad 0.000$

$(0.383) \quad(0.242)$

$-0.029$

(1.407)

0.000 *

(1.791)

$-0.532 * * *$

(3.708)

$-0.214^{*}$

(1.903)

$-0.228 *$

(1.801)

$-0.085$

(0.760)

$-0.064$

(0.462)

$\begin{array}{llll}0.241 * * * & 0.291 * * * & 0.130^{* *} & 0.199 * * *\end{array}$

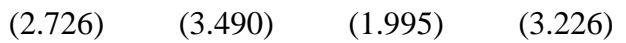

$\begin{array}{llll}-0.001 & -0.015 & -0.095 & -0.110\end{array}$

$\begin{array}{llll}(0.016) & (0.162) \quad(1.288) \quad \text { (1.498) }\end{array}$

$\begin{array}{llll}-0.008 & 0.021 & -0.256 * * & -0.187 *\end{array}$

$\begin{array}{llll}(0.064) \quad(0.161) & (2.398) \quad(1.798)\end{array}$

$\begin{array}{llll}0.158^{* *} & 0.140 * & 0.338 * * * & 0.321 * * *\end{array}$

$\begin{array}{llll}(2.159) & (1.924) \quad(6.116) \quad(5.859)\end{array}$

$\begin{array}{llll}-0.082 & -0.104 & -0.016 & -0.010\end{array}$

$\begin{array}{llll}(0.712) & (0.908) & (0.177) & (0.112)\end{array}$

$\begin{array}{llll}0.093 & 0.152 & 0.075 & 0.092\end{array}$

$\begin{array}{llll}(0.624) & (1.029) & (0.693) & (0.846)\end{array}$

$\begin{array}{llll}-0.260 & -0.236 & -0.128 & -0.143\end{array}$

$\begin{array}{llll}(1.366) & (1.241) \quad(1.104) \quad(1.235)\end{array}$

$\begin{array}{llll}0.035 & 0.038 & -0.070 & -0.049\end{array}$

$\begin{array}{llll}(0.250) & (0.276) & (0.760) & (0.531)\end{array}$

$\begin{array}{llll}0.034 & 0.022 & 0.034 & 0.039\end{array}$

$\begin{array}{llll}(0.244) & (0.159) & (0.372) & (0.426)\end{array}$

0.29 N $^{* *} \quad 0.268^{* *} \quad 0.193^{* *} \quad 0.181^{* *}$

$\begin{array}{llll}(2.192) & (1.970) \quad(2.112) \quad(1.987)\end{array}$

$0.495^{* * *} \quad 0.455^{* * *} \quad 0.420 * * * \quad 0.404 * * *$

(4.110)

$-0.217 * * * \quad(3.962)$

$\begin{array}{llll}-0.071 & -0.093 & -0.274 * * & -0.287 * * *\end{array}$

(2.520)

$0.113 * *$

(2.654)

$\begin{array}{lll}(3.834) & (2.069) \quad(1.892)\end{array}$

0.085

$(0.952)$

$-0.002$

0.108

(1.184)

(0.961)

(1.200) 


\begin{tabular}{|c|c|c|c|c|c|c|}
\hline Size 25 - 99 & $\begin{array}{l}0.032 \\
(0.352)\end{array}$ & $\begin{array}{l}0.054 \\
(0.597)\end{array}$ & $\begin{array}{l}-0.158 * \\
(1.787)\end{array}$ & $\begin{array}{l}-0.154 * \\
(1.722)\end{array}$ & $\begin{array}{l}-0.140 * * \\
(2.089)\end{array}$ & $\begin{array}{l}-0.127 * \\
(1.889)\end{array}$ \\
\hline Size 100 - 499 & $\begin{array}{l}-0.076 \\
(0.739)\end{array}$ & $\begin{array}{l}-0.047 \\
(0.451)\end{array}$ & $\begin{array}{l}-0.272^{* * *} \\
(2.596)\end{array}$ & $\begin{array}{l}-0.246 * * \\
(2.323)\end{array}$ & $\begin{array}{l}-0.240^{* * *} \\
(3.214)\end{array}$ & $\begin{array}{l}-0.232^{* * * *} \\
(3.076)\end{array}$ \\
\hline Size 500 plus & $\begin{array}{l}-0.105 \\
(0.955)\end{array}$ & $\begin{array}{l}-0.083 \\
(0.745)\end{array}$ & $\begin{array}{l}-0.117 \\
(1.055)\end{array}$ & $\begin{array}{l}-0.086 \\
(0.772)\end{array}$ & $\begin{array}{l}-0.271^{* * *} \\
(3.214)\end{array}$ & $\begin{array}{l}-0.254 * * * \\
(2.985)\end{array}$ \\
\hline Voluntarily Quit & $\begin{array}{l}0.235^{* *} \\
(2.003)\end{array}$ & $\begin{array}{l}0.237 * * \\
(2.017)\end{array}$ & $\begin{array}{l}0.300 * * * \\
(2.674)\end{array}$ & $\begin{array}{l}0.298 * * * \\
(2.656)\end{array}$ & $\begin{array}{l}0.415 * * * \\
(4.813)\end{array}$ & $\begin{array}{l}0.414^{* * *} \\
(4.794)\end{array}$ \\
\hline $\begin{array}{l}\text { Number of weeks not } \\
\text { employed in the last } \\
\text { year }\end{array}$ & $\begin{array}{l}0.001 \\
(0.401)\end{array}$ & $\begin{array}{l}0.001 \\
(0.186)\end{array}$ & $\begin{array}{l}0.003 \\
(0.896)\end{array}$ & $\begin{array}{l}0.002 \\
(0.689)\end{array}$ & $\begin{array}{l}0.004^{*} \\
(1.726)\end{array}$ & $\begin{array}{l}0.003 \\
(1.248)\end{array}$ \\
\hline Wave10 & $\begin{array}{l}-0.176^{* *} \\
(2.510)\end{array}$ & $\begin{array}{l}-0.162 * * \\
(2.302)\end{array}$ & $\begin{array}{l}-0.168 * * \\
(2.478)\end{array}$ & $\begin{array}{l}-0.161^{* *} \\
(2.376)\end{array}$ & $\begin{array}{l}-0.110 * * \\
(2.187)\end{array}$ & $\begin{array}{l}-0.096^{*} \\
(1.924)\end{array}$ \\
\hline Wave11 & $\begin{array}{l}-0.083 \\
(1.020)\end{array}$ & $\begin{array}{l}-0.013 \\
(0.144)\end{array}$ & $\begin{array}{l}-0.063 \\
(0.873)\end{array}$ & $\begin{array}{l}-0.050 \\
(0.617)\end{array}$ & $\begin{array}{l}-0.042 \\
(0.781)\end{array}$ & $\begin{array}{l}0.036 \\
(0.579)\end{array}$ \\
\hline $\begin{array}{l}\text { Log real } \\
\text { comparative wage }\end{array}$ & & $\begin{array}{l}-0.589 \\
(1.613)\end{array}$ & & $\begin{array}{l}-0.099 \\
(0.274)\end{array}$ & & $\begin{array}{l}-0.515 * * \\
(2.132)\end{array}$ \\
\hline Cut 1 & $\begin{array}{l}-4.33 * * * \\
(-5.22)\end{array}$ & $\begin{array}{l}-3.96 * * * \\
(-5.88)\end{array}$ & $\begin{array}{l}-2.89 * * * \\
(-3.30)\end{array}$ & $\begin{array}{l}-2.56^{* * *} \\
(-3.52)\end{array}$ & $\begin{array}{l}-4.13 * * * \\
(-7.11)\end{array}$ & $\begin{array}{l}-3.85 * * * \\
(-8.30)\end{array}$ \\
\hline Cut 2 & $\begin{array}{l}-3.70 * * * \\
(-4.50)\end{array}$ & $\begin{array}{l}-3.33 * * * \\
(-5.01)\end{array}$ & $\begin{array}{l}-2.24 * * \\
(-2.58)\end{array}$ & $\begin{array}{l}-1.92 * * \\
(-2.65)\end{array}$ & $\begin{array}{l}-3.50 * * * \\
(-6.07)\end{array}$ & $\begin{array}{l}-3.22 * * * \\
(-7.01)\end{array}$ \\
\hline Cut 3 & $\begin{array}{l}-2.98 * * * \\
(-3.64)\end{array}$ & $\begin{array}{l}-2.61 * * * \\
(-3.94)\end{array}$ & $\begin{array}{l}-1.54^{*} \\
(-1.77)\end{array}$ & $\begin{array}{l}-1.22^{*} \\
(-1.68)\end{array}$ & $\begin{array}{l}-2.87 * * * \\
(-4.98)\end{array}$ & $\begin{array}{l}-2.59 * * * \\
(-5.66)\end{array}$ \\
\hline Cut 4 & $\begin{array}{l}-2.63 * * * \\
(-3.22)\end{array}$ & $\begin{array}{l}-2.26 * * * \\
(-3.42)\end{array}$ & $\begin{array}{l}-1.19 \\
(-1.37)\end{array}$ & $\begin{array}{l}-0.87 \\
(-1.20)\end{array}$ & $\begin{array}{l}-2.47 * * * \\
(-4.30)\end{array}$ & $\begin{array}{l}-2.19 * * * \\
(-4.81)\end{array}$ \\
\hline Cut 5 & $\begin{array}{l}-1.67 * * \\
(-2.05)\end{array}$ & $\begin{array}{l}-1.30 * * \\
(-1.98)\end{array}$ & $\begin{array}{l}-0.33 \\
(-0.38)\end{array}$ & $\begin{array}{l}-0.01 \\
(-0.02)\end{array}$ & $\begin{array}{l}-1.52^{* *} \\
(-2.65)\end{array}$ & $\begin{array}{l}-1.24^{* *} \\
(-2.73)\end{array}$ \\
\hline Cut 6 & $\begin{array}{l}0.48 \\
(0.59)\end{array}$ & $\begin{array}{l}0.84 \\
(1.28)\end{array}$ & $\begin{array}{l}1.69 \\
(1.95)\end{array}$ & $\begin{array}{l}2.00 \\
(2.76)\end{array}$ & $\begin{array}{l}0.52 \\
(0.91)\end{array}$ & $\begin{array}{l}0.79 * \\
(1.75)\end{array}$ \\
\hline Log Likelihood & -2544 & -2550 & -2505 & -2515 & -4727 & -4739 \\
\hline Observations & 1874 & 1874 & 1830 & 1830 & 3490 & 3489 \\
\hline
\end{tabular}

Absolute value of $\mathrm{z}$ statistics in parentheses

* significant at $10 \%$; ** significant at $5 \%$; *** significant at $1 \%$ 
Table 4: Variable Definitions

Overall Job Satisfaction Overall Job Satisfaction

Gender

Log usual real gross hourly wage

Log usual hours

Job Tenure

Tenure Squared

Age

Age square

University degree

Vocational qualifications

A - levels plus

$\mathrm{O}$ - levels plus

Commercial

Apprentice

Married

Mortgage house

Paid outright house

White

Promotion

Public Sector

Works mornings only

Works evenings or

nights

Variable shift patterns

Fair health

Good health

Excellent health

Trade union member

Temporary Job

Incremental pay

Part Time

Travel Time
Gender of respondent

Log of average real hourly earnings

Log of usual hours

Job Tenure in months

Job Tenure in months squared

Age at date of interview

Age at date of interview squared

Highest qualification is

postgraduate \& first degree

Highest qualification is

Vocational degrees : Teaching,

Nursing hnd other

Highest qualification is A-levels

or equivalent

Highest qualification is O-levels or equivalent

or

Highest qualification is

Commercial and apprenticeships

Married

Owns home with mortgage

Owns home outright

Ethnic background of individual promotion opportunities in current job

Public Sector

Works mornings only

Works evenings or nights

Variable shift patterns

Fair health

Good health

Excellent health

Trade Union Member

Individual has a temporary Job

Individual's pay includes annual increments

Individual is employed Full-

Time

Minutes spent travelling to work
$1=$ not satisfied at all

4= not satisfied /dissatisfied

7 = completely satisfied

$1=$ Males

$0=$ Females

1 if the individual's highest qualification is postgraduate \& first degree; 0 otherwise.

1 if the individual's highest qualification is Vocational degrees :

Teaching, Nursing hnd other

1 if the individual's highest qualification is A-levels or equivalent 1 if the individual's highest qualification is O-levels or equivalent 1 if the individual's highest qualification is Commercial and apprenticeships

1 if the individual is married

1 if the individual's home is owned with a mortgage; 0 otherwise 1 if the individual's home is owned outright; 0 otherwise

1 if individual is white; 0 otherwise 1 if the individual has promotion opportunities in current job; 0 otherwise

1 if the individual is employed in the public sector; 0 otherwise

1 if the individual works in the mornings only; 0 otherwise

1 if the individual works evenings or nights; 0 otherwise

1 if the individual has variable shift patterns; 0 otherwise

1 if individual has fair health; 0

otherwise

1 if individual has good health; 0

otherwise

1 if individual has excellent health; 0

otherwise

1 if the individual is a trade union member; 0 otherwise

1 if the individual has a temporary job; 0 otherwise

1 if the individual's pay includes annual increments; 0 otherwise 1 if the individual is employed FullTime; 0 otherwise 
Size 25 - 99

Size 100 - 499

Size 500 plus

Energy and Water

Manufacturing

Construction

Distribution, Hotels etc

Transport, communications

Banking and Finance

Public Administration

Education and Health

Other

Professional

Occupations

Associate professional and technical

Administrative and Secretarial

Skilled Trades

Service Occupations

Process Plant and Machine Operatives

Elementary

Occupations

Voluntarily Quit

Number of weeks not employed in the last year

Wave10
25-99 employees

size499

size500

Energy and Water

Manufacturing

Construction

Distribution, Hotels etc

Transport, communications

Banking and Finance

Public Administration

Education and Health

Other

Professional Occupations

Associate professional technical

Administrative and Secretarial

Skilled Trades

Service Occupations

Process Plant and Machine Operatives Elementary Occupations

Voluntarily Quit

Number of weeks not employed in the last year

wave10
1 if individual is employed in an workplace with between 25 and 99 employees; 0 otherwise

1 if individual is employed in an workplace with between 25 and 99 employees; 0 otherwise

1 if individual is employed in an workplace with between 25 and 99 employees; 0 otherwise 1 if individual is employed in an workplace in the energy and water sector; 0 otherwise

1 if individual is employed in an workplace in the manufacturing sector; 0 otherwise

1 if individual is employed in an workplace in the construction sector; 0 otherwise

1 if individual is employed in an workplace in the distribution and hotel sector; 0 otherwise

1 if individual is employed in an workplace in the transport and communications sector; 0 otherwise 1 if individual is employed in an workplace in the banking and finance sector; 0 otherwise

1 if individual is employed in an workplace in the public administration sector; 0 otherwise

1 if individual is employed in an workplace in the education and health; 0 otherwise

1 if individual is employed in an workplace in the other sector; 0 otherwise

1 if individual is employed in Professional Occupations; 0 otherwise 1 if individual is employed in

and Associate professional and technical; 0 otherwise

1 if individual is employed in Administrative and Secretarial; 0 otherwise

1 if individual is employed in Skilled Trades; 0 otherwise

1 if individual is employed in Service Occupations; 0 otherwise

1 if individual is employed in Process

Plant and Machine Operatives; 0

otherwise

1 if individual is employed in Elementary Occupations; 0 otherwise 1 if the individual voluntarily quit previous job; 0 otherwise

1 if the observation is in wave $10 ; 0$ otherwise 


$\begin{array}{ll}\text { Wave11 } & \text { wave11 } \\ \text { Rest of England } & \text { Region is Rest of England } \\ \text { London and South East } & \begin{array}{l}\text { Region is London and South } \\ \text { East } \\ \text { Scotland }\end{array} \\ \text { Region is Scotland }\end{array}$

1 if the observation is in wave 10; 0 otherwise

1 if the individual lives in the England outside of London and the South East; 0 otherwise

1 if the individual lives in London and the South East; 0 otherwise

1 if the individual lives in Scotland; 0 otherwise 
Figure One: Mean Overall Job Satisfaction by Region, Waves 1-11

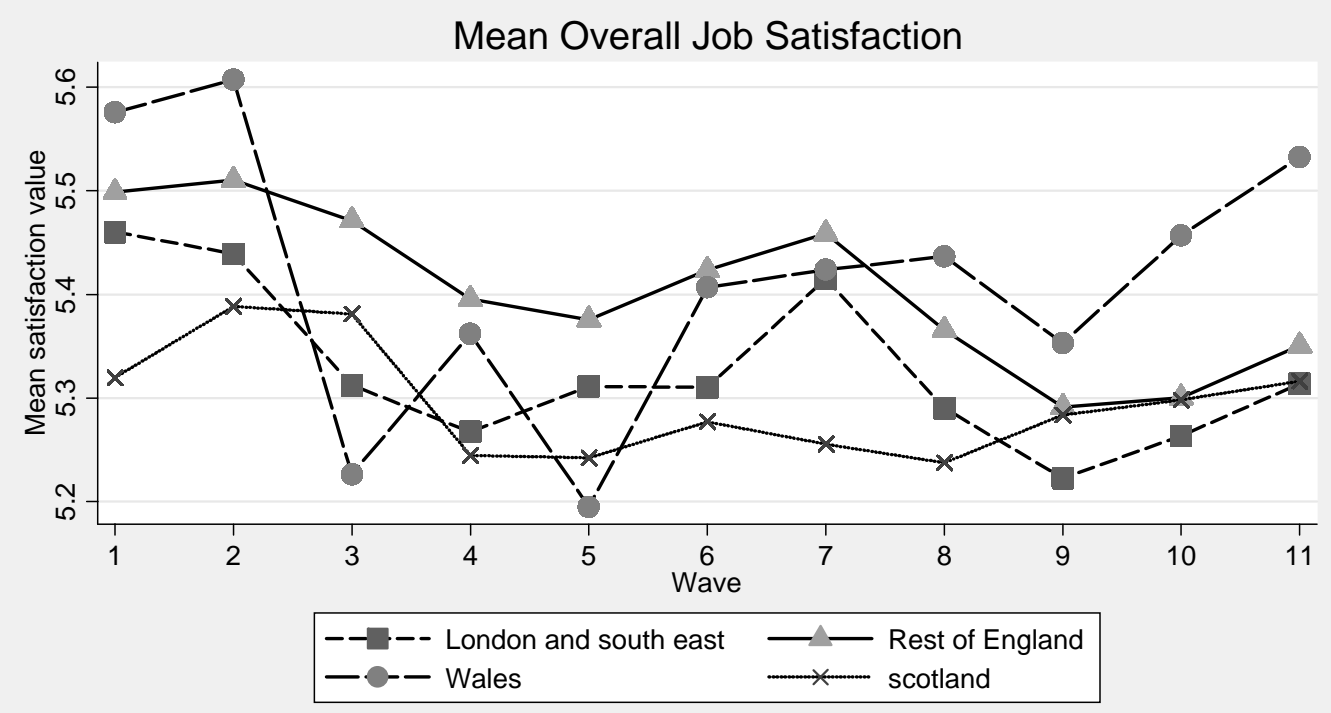

Source: British Household Panel Survey

Figure Two: Mean Satisfaction with Pay by Region, Waves 1-11

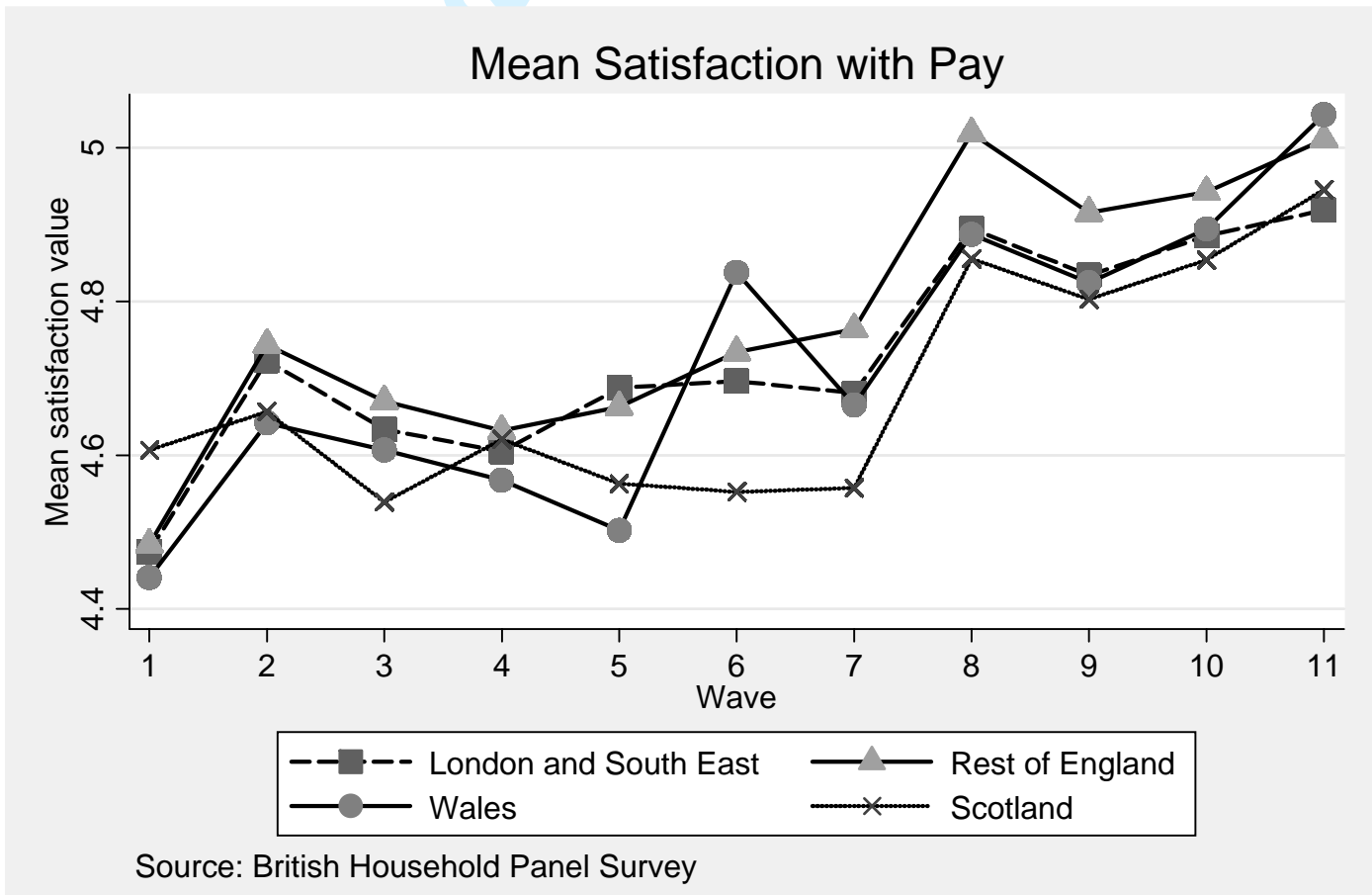


Figure Three: Mean Overall Life Satisfaction by Region, Waves 6-10

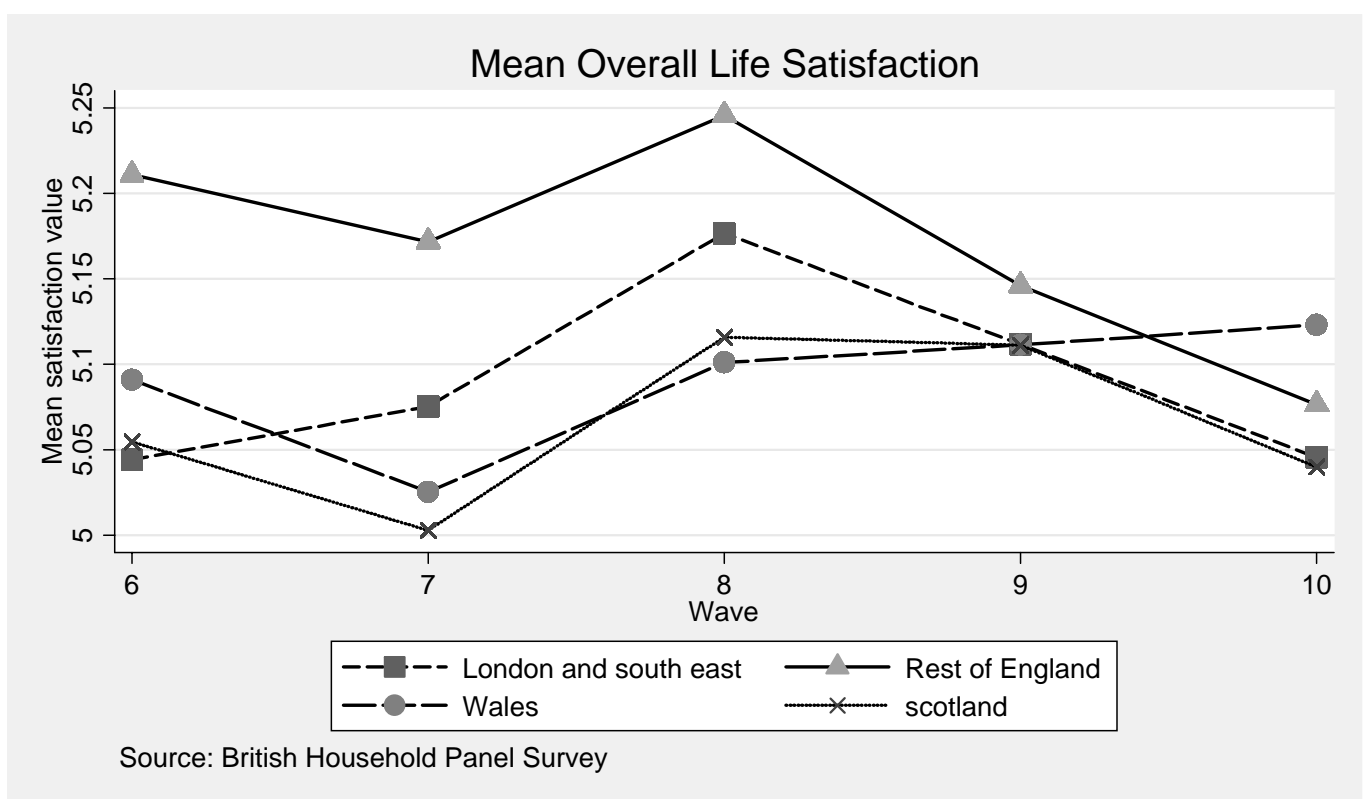


Figure Four: Mean Overall Life Satisfaction by Level of Job Satisfaction, Waves 610

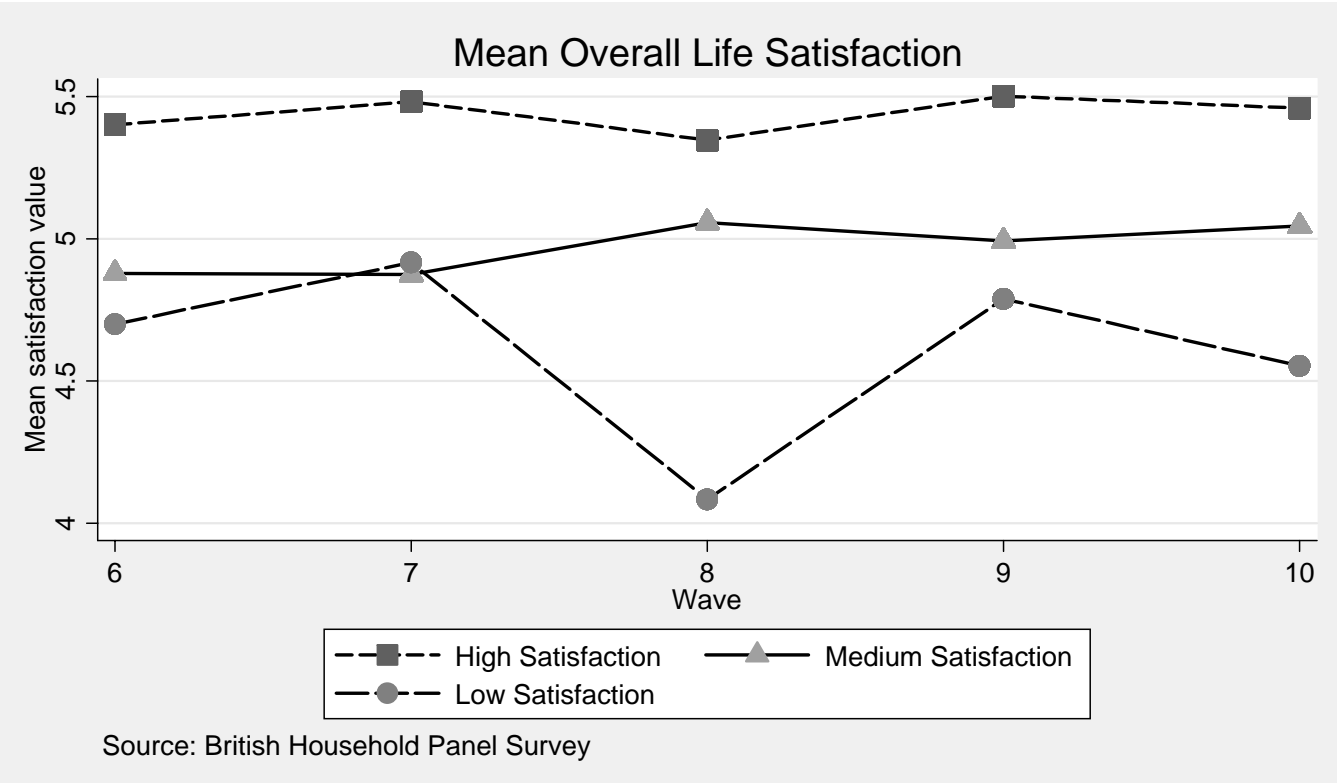

Figure Five: Mean Overall Life Satisfaction Workers only, Waves 6-10

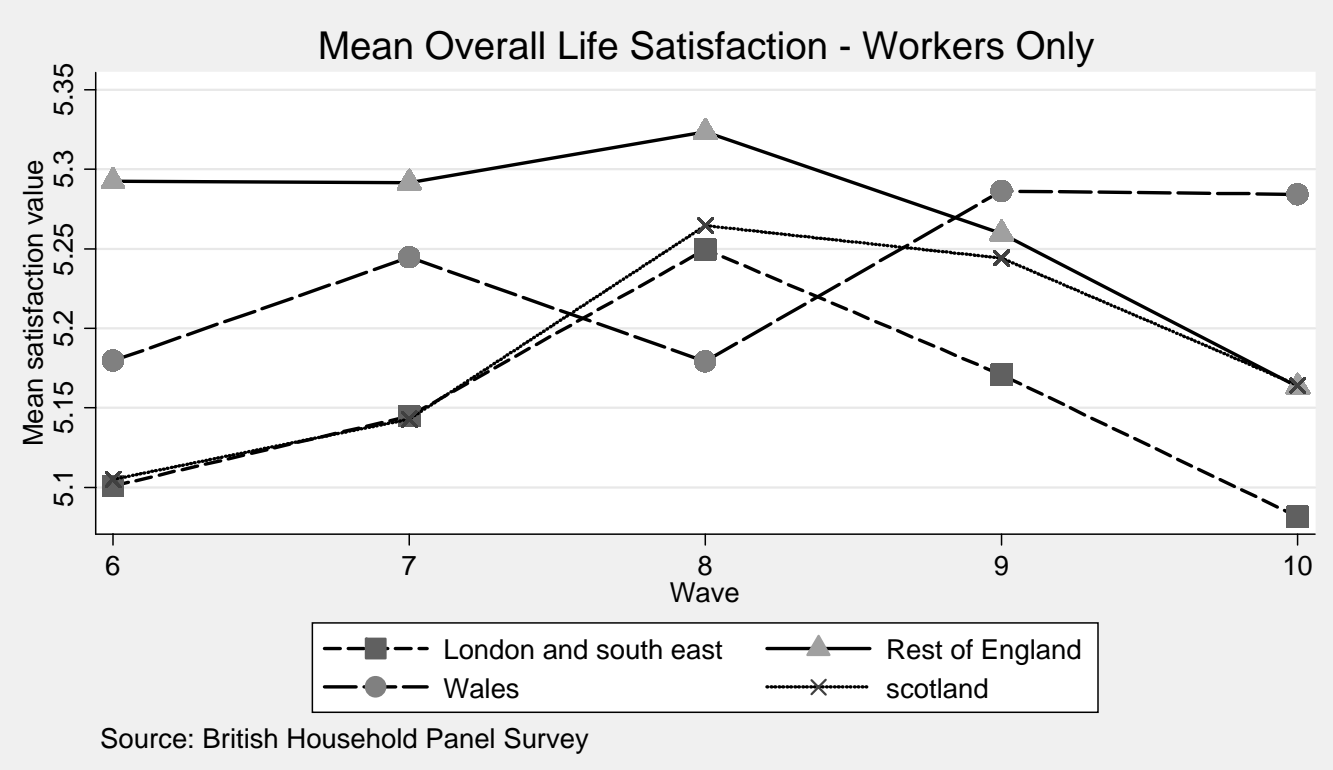


Figure Six: Mean Overall Life Satisfaction in Wales by Employment Status, Waves 6-10

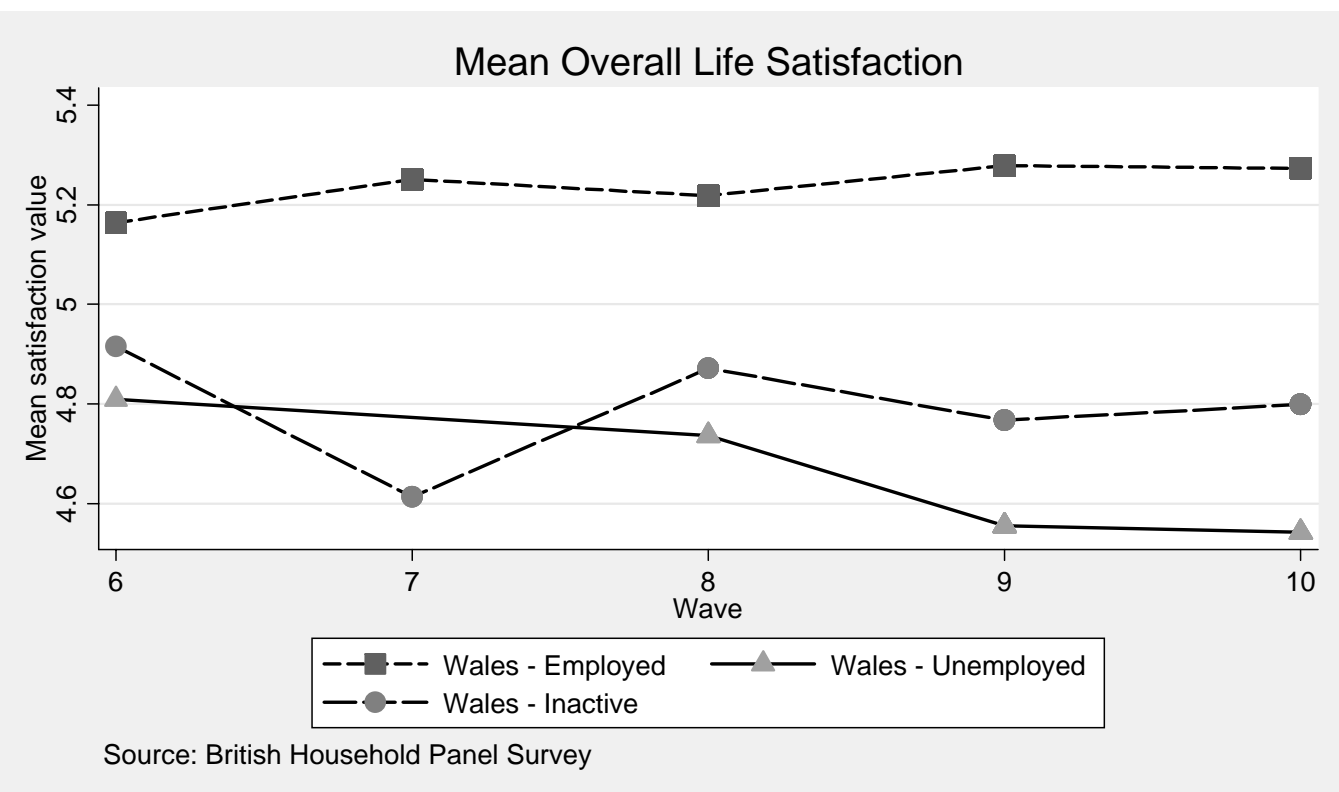




\section{Acknowledgements}

Financial support from the European Social Fund is gratefully acknowledged. 


\section{REFERENCES}

Akerlof G.A., Rose A.K., and Yellen J.L., (1988) Job Switching and Job Satisfaction in the U.S. Labour Market, Brookings Papers on Economic Activity, 2, 493-582.

Baxter J.L. (1973) Inflation in the Context of Relative Deprivation and Social Justice, Scottish Journal of Political Economy, 20, 263-282.

Baxter J.L. (1993) Behavioural Foundations in Economics, MacMillan Press.

Belfield C.R. and Harris R.D.F., How Well Do Theories of Job Matching Explain Variations in Job Satisfaction Across Education Levels? Evidence for U.K. Graduates, Applied Economics, Vol. 34, 2002, pp 535-548

Bender K and Sloane P.J. (1998) Job Satisfaction, Trade Unions and Exit-Voice Revisited, Industrial and Labor Relations Review, 51, 222-240.

Brown D. and McIntosh S., Job Satisfaction in the Low Wage Service Sector, Applied Economics, Vol. 35, 2003, pp 1241-1254

Clark A.E., Job Satisfaction in Britain, British Journal of Industrial Relations, Vol. 34, 1996, pp 189-217

Clark A.E. (2001) What really Matters in a Job? Hedonic Measurement Using Quit Data, Labour Economics, 8(2), 223-242.

Clark A.E. and Oswald A.J., Satisfaction and Comparison Income, Journal of Public Economics, Vol. 61, 1996, pp 359-81.

Clegg C.W. (1983) Psychology of Employee Lateness, Absence and Turnover: Methodology, a Critique and an Empirical Study, Journal of Applied Psychology, 68, 88-101.

Diaz-Serrano L. and Cabral Vieira Jose A., Low Pay, Higher Pay and Job Satisfaction within the European Union: Empirical Evidence from Fourteen Countries, IZA Discussion Paper No. 1558, Bonn, April, 2005

Drinkwater S. and Blackaby D. (2004) Migration and Labour Market Differences: The Case of Wales, paper presented at the Royal Economics Society Conference, University of Wales Swansea.

Drinkwater S and Ingram P. (2003) Have Industrial Relations in the UK Really Improved?, Unpublished manuscript, University of Surrey, Guildford.

Easterlin R. (1974) Does Economic Growth Improve the Human Lot? in David P, and Reder M, editors, Nations and Households in Economic Growth: Essays in Honor of Moses Abramovitz, Academic Press, New York,

Frank R.F. (1985) Choosing the Right Pond, Oxford University Press, Oxford and New York. 
Freeman R.B. (1978) Job Satisfaction as an Economic Variable, American Economic Review, 68, 135-141

Gazioglu S. and Tansel A., Job Satisfaction in Britain: Individual and Job Related Factors, Applied Economics, Vol. 38, 2006, pp 1163-1171

Green F. and Tsitsianis N., An Investigation of National Trends in Job Satisfaction in Britain and Germany, British Journal of Industrial Relations, Vol. 43, No. 3, September, 2005, pp 401-429

Hamermesh D.S. (1977) Economic Aspects of Job Satisfaction, in Ashenfelter O.E. and Oates W.E., editors, Essays in Labor Market Analysis, John Wiley, New York, pp 53-72

Idson T.L., Establishment Size, Job Satisfaction and the Structure of Work, Applied Economics, Vol. 22, 1990, pp 1007-1018

Kristensen N. and Westergard-Nielsen N. (2004) Does Job Satisfaction Lead to Job Mobility?, IZA Discussion Paper No. 1026, February

Leontaridi R. M., Sloane P. J. and Jones R. J. (2004). Are Low Paid Jobs of Low Ability? Some British Evidence. WELMERC Discussion Paper, no. 2004-02, University of Wales Swansea,

Lydon R. and Chevalier A., (2002) Estimates of the Effects of Wages on Job Satisfaction,' unpublished manuscript, Centre for Economic Performance, London School of Economics.

Mangione J. W. and Quinn R. P., (1975) 'Job Satisfaction, Counter-productive Behaviour and Drug Use in Work. Journal of Applied Psychology, 60, 114 - 116.

Major B and Forcey B., (1985) Social Comparisons and Pay Evaluations; Preferences for Same Sex and Same Job Wage Comparison. Journal of Experimental Social Psychology, vol 21, pp 243-252

Meng R., 'The Relationship between Unions and Job Satisfaction, Applied Economics, Vo. 22, 1990, pp 1635-1648

Oswald A. J., (1997) Happiness and Economic Performance, Economic Journal 107, no. $445,1815-1831$

Rees A., (1993) The Role of Fairness in Wage Determination, Journal of Labor Economics, 11, 243-252.

Sloane P.J., and Williams H., (2000) Job Satisfaction, Comparison Earnings and Gender Labour, 14(3), 473 - 501. 
Sousa-Poza A. and Sousa-Poza A.A., Well-Being at Work: A Cross National Analysis of the Levels and Determinants of Job Satisfaction, Journal of SocioEconomics, Vol. 29, 2000, pp 517-538

Sousa-Poza A. and Sousa-Poza A.A., Taking Another Look at the Gender/Job Satisfaction Paradox, Kyklos, Vol. 53, 2000, pp 135-152

Sousa-Poza A. and Sousa-Poza A.A., (2003), 'Gender Difference in Job Satisfaction in Great Britain, 1991-2000, Permanent or Transitory?’, Applied Economics Letters, $10,691-694$. 